\title{
CAMBIOS NORMATIVOS EN LA DIGITALIZACIÓN DEL TRABAJO: COMENTARIO A LA “LEY RIDER" Y LOS DERECHOS DE INFORMACIÓN SOBRE LOS ALGORITMOS ${ }^{1}$
}

\author{
Adrián Todolí Signes \\ Profesor Contratado Doctor \\ Departamento de Derecho del Trabajo y de la Seguridad Social de la \\ Universidad de Valencia
}

\begin{abstract}
La conocida como "ley Rider", fruto del dialogo social entre patronal, sindicatos y Gobierno, regula dos cuestiones. De un lado, una nueva DA23 ET, que incorpora una presunción de laboralidad de los repartidores. De otro lado, la obligación de las empresas que usen algoritmos que puedan tener efectos sobre las condiciones laborales de informar de ello a los representantes de los trabajadores. En este trabajo se analiza el alcance y trascendencia de ambas disposiciones. Así, se argumenta que ambas disposiciones aportan novedades que, aunque aparentemente se limitan a simples aclaraciones interpretativas de normas ya existentes, son un primer paso hacia la regulación a nivel normativo de los efectos de la digitalización en el trabajo. El trabajo concluye que la norma viene a determinar que las nuevas posibilidades tecnológicas no son más que medios de producción por lo que se ejerce una actividad económica y, por ello, las normas laborales deben serles de aplicación.
\end{abstract}

The so-called "Rider law", the result of social dialogue between employers, unions and the government, regulates two issues. On the one hand, a new DA23 Labour Estatute, which incorporates a presumption of employment contract for delivery drivers on platforms. On the other hand, the obligation of companies that use algorithms that may have an effect on working conditions to inform the workers' representatives. This paper analyzes the scope and significance of both provisions. Thus, it is argued that both provisions provide novelties that, although apparently limited to simple interpretative clarifications of already existing rules, are a first step towards the regulation of the effects of digitalization on the workplace at the regulatory level. The paper concludes that the regulation determines that the new technological possibilities are nothing more than means of production through which an economic activity is exercised and, therefore, the labor rules should be applicable to them.

\footnotetext{
${ }^{1}$ Investigación parte del Proyecto de I+D+i Retos MICINN "Derechos y garantías frente a las decisiones automatizadas en entornos de inteligencia artificial, IoT, big data y robótica" (PID2019-108710RB-I00, 2020-2022).
} 
Title: legal changes related to employment digitalisation: comment regarding the "Rider Act" and the rights of information related to algorithms.

Palabras clave: "Ley Rider", trabajo en plataformas, plataformas digitales, algoritmos, derechos de información de los representantes de los trabajadores.

Keywords: Rider Act, platform work, digital platforms, algorithm, information rights of workers' representatives.

IUSLabor 2/2021, ISSN 1699-2938, p. 28-65

DOI. 10.31009/IUSLabor.2021.i02.2

Fecha envío: 17.5.2021 | Fecha aceptación: 26.5.2021

\section{Sumario}

1. Los primeros pasos de unos legisladores inseguros

2. Presunción de laboralidad en el ámbito de las plataformas digitales de reparto

2.1. Origen jurisprudencial de la "nueva" presunción

2.2. Contenido de la "nueva" presunción

2.2.1. Presunción e inversión de la carga de la prueba

2.2.2. Reducción de intensidad del elemento de dependencia jurídica

2.3. Trascendencia de la nueva presunción

3. El deber de transparencia algorítmica

3.1. Derecho de información de los representantes de los trabajadores sobre los algoritmos

3.2. Concepto de algoritmo

3.3. Comparativa entre los nuevos derechos de información y la regulación europea ya existente

3.4. Contenido de la información a entregar

3.5. Derechos de información ya existentes que afectan a los algoritmos

3.6. Trascendencia del nuevo derecho de información

4. Entrada en vigor a los tres meses de su publicación

5. Valoración final 


\section{Los primeros pasos de unos legisladores inseguros}

El trabajo en plataformas y los efectos de la inteligencia artificial están copando, en los últimos años, el interés de la doctrina y del legislador laboral en muchos países. Así, informes de instituciones como la OIT $(2021)^{2}$, y la Comisión Europea (2020 ${ }^{3}$ y 2018 $)$, han señalado el fuerte impacto que tiene este tipo de empleo en el empeoramiento de las condiciones laborales, así como su rápida expansión en todas las economías desarrolladas y en vías de desarrollo ${ }^{5}$. Este hecho ha levantado un interés inusitado por parte de los legisladores.

Así, California, en 2019, reguló la Ley AB5, en la que se incorporaba el test judicial de laboralidad "ABC", que moderniza el concepto de trabajador y lo adapta a las nuevas formas de trabajar ${ }^{6}$, con objeto de evitar la "deslaboralización" en la nueva tipología de empresas. Una norma que, un año después de su aprobación, quedó seriamente limitada al ser aprobada por referéndum la "Proposición 22", que la hace inaplicable a ciertos trabajadores de plataformas como los de Uber y $\mathrm{Lyft}^{7}$.

También, en noviembre de 2019, Italia aprobó una nueva ley con objeto de regular el trabajo en plataformas ${ }^{8}$. La ley introduce dos regímenes legales. Por una parte, cubre a los "trabajadores que prestan personalmente servicios organizados por el cliente a través de plataformas digitales". Por otro lado, se establece una protección laboral a los "trabajadores autónomos que reparten bienes a través de medios de dos ruedas en áreas urbanas".

\footnotetext{
${ }^{2}$ OIT, The role of digital labour platforms in transforming the world of work, Ginebra, 2021.

${ }^{3}$ Urzì Brancati, María Cesira, Pesole, Ana Rosa, Fernández-Macías, Enrique, New evidence on platform workers in Europe. Results from the second COLLEEM survey, EUR 29958 EN, Publications Office of the European Union, Luxembourg, 2020.

${ }^{4}$ Pesole, Annarosa, Urzí Brancati, Maria Cesira, Fernández-Macías, Enrique, Biagi, Federico, GonZÁlez VÁzquez, Ignacio, Platform Workers in Europe, EUR 29275 EN, Publications Office of the European Union, Luxembourg, 2018.

${ }^{5}$ Para el caso de España, ver Fundación Felipe González, Huella Digital: La plataformización del trabajo en Europa, 2019.

${ }^{6}$ Todolí SIGNES, Adrián "El ámbito subjetivo de aplicación del Estatuto de los Trabajadores ante las nuevas formas de trabajo: la nueva ley de California (AB5)", TyD, $\mathrm{n}^{\circ} 66,2020$.

${ }^{7}$ Conger, Kate, "Uber and Lyft Drivers in California Will Remain Contractors", New York Times, https://www.nytimes.com/2020/11/04/technology/california-uber-lyft-prop-22.html.

${ }^{8}$ Law No. 128/2019 amending Decree 101/2019 on urgent measures for the protection of work.

${ }^{9}$ ALOISI, Antonio, (2020), "A fascinating chapter in the "gig" saga. How to deliver decent work to platform workers in Italy?", Mutual learning Programme, DG Employment, Social Affairs and Inclusion, European Union, 2020 ;

https://ec.europa.eu/social/main.jsp?catId=89\&furtherNews=yes\&newsId=9746\&langId=en\#navItem-1
} 
A nivel europeo, la Directiva 2019/1152 del Parlamento Europeo y del Consejo, de 20 de junio de 2019, relativa a unas condiciones laborales transparentes y previsibles en la Unión Europea, pretendió incorporar una definición de trabajador europeo que incluyera a los trabajadores de plataformas para armonizar el debate en la UE. Sin embargo, esa parte de la Directiva decayó, limitándose a recordar que su aplicabilidad depende del concepto del TJUE sobre trabajador ${ }^{10}$.

No obstante, de nuevo, en el marco europeo, se está analizando la posibilidad de extender los derechos de negociación colectiva a todos los trabajadores de plataformas ${ }^{11}$. Así, la Comisión europea, en junio de 2020, emitió una consulta pública, como primer paso para la elaboración de una propuesta sobre la regulación de los derechos de negociación colectiva de los autónomos individuales incluyendo, específica y especialmente, a los trabajadores de plataformas ${ }^{12}$.

En lo que respecta a la materia reguladora de los algoritmos e inteligencia artificial, a pesar de lo reciente que es el RGPD, en cuyo artículo 22 se regula, específicamente, el uso de la IA de algoritmos para la toma de decisiones automatizada y el perfilado automático, la UE, ha publicado en abril del 2021, un "borrador" de Reglamento sobre su uso para tomar decisiones que puedan afectar, entre otros, a los trabajadores. Un reglamento que, como ha criticado la doctrina, a pesar de calificar de "riesgo alto" para los trabajadores determinadas actuaciones de los algoritmos, no ha incluido derechos para los representantes de los trabajadores en materia de información, consulta ni negociación colectiva sobre los mismos ${ }^{13}$.

En este marco de alto interés legislativo internacional, pero con normas de limitados efectos, se enmarca la nueva regulación española. Ciertamente, esta normativa, que es el resultado de un acuerdo entre el Gobierno, la patronal y los agentes sociales más representativos, comenzó su proceso de elaboración con unas mayores expectativas. Los

\footnotetext{
${ }^{10}$ Sobre este concepto ver, CABEZA Pereiro, Jaime, El ámbito del trabajo subordinado y del trabajo autónomo en el Derecho de la Unión Europea, Bomarzo, 2020; TODOLÍ SigNES, Adrián, "El concepto de trabajador en el Derecho de la Unión Europea y su aplicación a las nuevas realidades económicas: Comentario al auto motivado del TJUE en el caso de los riders", $T y D, \mathrm{n}^{\circ} 70,2020$. SÁNCHEZ-URÁN AZAÑA, Yolanda, "Concepto de trabajador y economía digital. Respuesta en el contexto internacional y en el derecho de la UE", Revista de Derecho Social y Empresa, n 14, 2021.

${ }^{11}$ Sobre el tema ver las interesantes aportaciones de GoERLICH PESET Jose Maria, LÓPEZ TERRADA, Eva, "Cambios productivos, reestructuraciones y negociación colectiva", Gaceta Sindical, 32, 2019.

${ }^{12}$ ver Countouris, Nicola, De Stefano, Valerio, y Lianos, Ioannis, "The EU, competition and worker's Rights", Center for Law, Economics and Society, n 2, 2021.

${ }^{13}$ De Stefano, Valerio, "The EU Proposed Regulation on AI: a threat to labour protection?", Regulation for Globalization, 2021, http://regulatingforglobalization.com/2021/04/16/the-eu-proposed-regulation-onai-a-threat-to-labour-protection/
} 
primeros borradores de la norma contemplaban un ámbito de aplicación mucho mayor (que incluía todo tipo de plataformas digitales en todos los sectores) que el finalmente aprobado: que incluye solamente las plataformas de reparto. También, en sus inicios, la normativa acogía, por solicitud de los sindicatos, la creación de un registro obligatorio de plataformas digitales de carácter público que decayó durante el proceso negociador.

Así, la normativa, que ha sido aprobada por el Gobierno mediante el Real Decreto-ley 9/2021, de 11 de mayo, para garantizar los derechos laborales de las personas dedicadas al reparto en el ámbito de plataformas digitales, incluye dos disposiciones; ii) la primera no se limita al trabajo en plataformas, sino que se enmarca dentro de los efectos de los algoritmos y la inteligencia artificial en el trabajo en cualquier empresa que use estos modernos sistemas tecnológicos. Concretamente, se establece el derecho de información de los representantes de los trabajadores sobre los parámetros, reglas e instrucciones en los que se basan los algoritmos o sistemas de inteligencia artificial que afectan a la toma de decisiones que pueden incidir en el trabajo; i) la segunda es una presunción de laboralidad en el ámbito de las plataformas digitales de reparto;

Ambas disposiciones entrarán en vigor tres meses después de su publicación. Un tiempo que se entiende más que razonable para que las empresas realicen las adaptaciones que se consideren necesarias para su cumplimiento, sobre todo, porque como ahora se verá, ninguna de las dos disposiciones incorpora nada realmente nuevo al ordenamiento jurídico español, sino que se limitan a declarar o clarificar dos obligaciones que las empresas ya tenían con anterioridad derivado de preceptos normativos de existencia previa.

\section{Presunción de laboralidad en el ámbito de las plataformas digitales de reparto}

\subsection{Origen jurisprudencial de la “nueva” presunción}

En relación con la presunción de laboralidad de los repartidores en plataformas digitales este RDL 9/2021 incorpora al Estatuto de los trabajadores de una nueva Disposición Adicional 23 que establece lo siguiente:

Disposición adicional vigesimotercera. Presunción de laboralidad en el ámbito de las plataformas digitales de reparto

Por aplicación de lo establecido en el artículo 8.1, se presume incluida en el ámbito de esta ley la actividad de las personas que presten servicios retribuidos consistentes en el reparto o distribución de cualquier producto de consumo o mercancía, por parte de empleadoras que ejercen las facultades empresariales 
de organización, dirección y control de forma directa, indirecta o implícita, mediante la gestión algorítmica del servicio o de las condiciones de trabajo, a través de una plataforma digital.

Esta presunción no afecta a lo previsto en el artículo 1.3 de la presente norma.

Con objeto de comentar esta nueva disposición se debe partir, irremediablemente, de la jurisprudencia existente en materia de concepto de trabajador por dos motivos. En primer lugar, porque la jurisprudencia del Tribunal Supremo viene entendiendo, previamente a esta regulación, que el artículo 8.1 ET ya recoge una presunción de laboralidad. De esta forma, en los últimos años ha habido una revitalización del uso de la presunción de laboralidad por parte de los Tribunales con objeto de facilitar la prueba de la laboralidad a los Trabajadores. De este modo, todo aquel que preste servicios retribuidos de forma personal tendrá que entenderse laboral salvo que el empresario pruebe que el contrato que une a las partes es de naturaleza distinta al contrato de trabajo. Esta no es una cuestión material, que modifique los requisitos de aplicación del contrato de trabajo, sino meramente una forma de determinar que es el empresario el que debe probar, si así le interesa, la exclusión de un prestador de servicios del contrato de trabajo.

En segundo lugar, el Tribunal Supremo también ha modificado los requisitos del contrato de trabajo con su interpretación. En efecto, en las últimas décadas, pero con especial incidencia en los últimos años, el máximo órgano judicial ha flexibilizado el requisito de dependencia jurídica para entender que, determinada relación contractual, se encuentra enmarcada dentro del contrato de trabajo. Como he tenido ocasión de analizar en profundidad en otros lugares ${ }^{14}$, el máximo Tribunal, parece haber abandonado $-\mathrm{O}$ al menos reducido a la mínima expresión- la cuestión de la dependencia jurídica o el control como forma de identificar a los trabajadores.

En este sentido, en multitud de ocasiones, el Tribunal Supremo ha entendido que existe contrato de trabajo, aunque el trabajador tenga libertad para elegir sus propios horarios o su jornada de trabajo ${ }^{15}$. En el mismo sentido, también se ha entendido que puede existir

\footnotetext{
${ }^{14}$ TODOLí SIGNES, Adrián, "Plataformas digitales y concepto de trabajador: una propuesta de interpretación finalista", Lan harremanak, no 41, 2019.

${ }^{15}$ STS 20 de enero de 2015 (rec. 587/2014) -caso de limpiadores de escaleras-; STS 20 de julio de 2010 (rec. 3344/2009) -limpiador de oficinas-; STS 22 de enero de 2008 (rec. 626/2007) -Transportistas con vehículo propio-; STS de 30 de abril de 2009 (1701/2008) -recogedor productos higiénicos-; STS de16 de julio de 2010 (rec. 3391/2009 y 2830/2009) de 19 de julio 2010 (rec. 1623/2009 y 2233/2009) -artistas y dobladores-; STS 3 de mayo de 2005 (rec. 2606/2004) -abogados-; STS 21 de junio de 2011 (rec. 2355/2010) -subagentes de seguros-; STS de 14 de julio de 2016 (rec. 539/2015) -Agentes de Seguros-; STS DE 16 de noviembre 2017 (rec 2806/2015) -traductores-; STS 8 de febrero de 2018 (REC. 3205/2015) -Montadores de ascensores-.
} 
laboralidad, aunque no haya instrucciones por parte del empresario ${ }^{16}$. E incluso se entiende que la facultad del trabajador para rechazar trabajos encargados por el empresario no excluye automáticamente la laboralidad ${ }^{17}$. Tampoco la inexistencia de lugar de trabajo asignado por el empresario (trabajo online desde el lugar elegido libremente por el trabajador) ha evitado la calificación de laboral del trabajador ${ }^{18}$. Por tanto, los clásicos indicios de la dependencia como son la fijación de horarios, las instrucciones dictadas por el empresario, el control del trabajo, así como la elección empresarial del lugar de prestación de servicios, parecen servir para confirmar la laboralidad, pero no para excluirla cuando se dan otros indicios.

En fin, ambos motivos -presunción de laboralidad y flexibilización en la aplicación del requisito de dependencia jurídica- son esgrimidos por el Tribunal Supremo en la Sentencia que califica como trabajador laboral un repartidor de Glovo (STS de 25 de septiembre de 2020 (rec. 4746/2019)) ${ }^{19}$ y que inspira la regulación aquí comentada.

${ }^{16}$ STS 20 de julio de 2010 (rec. 3344/2009) -limpiador de oficinas-; STS 22 de enero de 2008 (rec. 626/2007) -Transportistas con vehículo propio-; STS de 30 de abril de 2009 (1701/2008) -recogedor productos higiénicos-; STS de 16 de noviembre 2017 (rec 2806/2015) -Traductores-; STS 8 de febrero de 2018 (REC. 3205/2015) -Montadores de ascensores-.

${ }^{17}$ STS de 16 de noviembre 2017 (rec 2806/2015) -Traductores-; STS de 16 de julio de 2010 (rec. 3391/2009 y 2830/2009) de 19 de julio 2010 (rec. 1623/2009 y 2233/2009) -artistas y dobladores-.

${ }^{18}$ STS 29 de octubre de 2019 (rec. 1338/2017).

${ }^{19}$ Ver comentarios en Monereo Pérez, José Luis, y MARTín MuÑoz, María Rosa, "La laboralidad de quienes prestan servicios a través de plataformas digitales", Revista de Jurisprudencia Laboral, $\mathrm{n}^{\circ}$ 9, 2020; Álvarez Alonso, Diego, y MARTínez Moreno, Carolina, "Trabajo y plataformas digitales primera sentencia del Tribunal Supremo a propósito de un repartidor de Glovo", TyD, $\mathrm{n}^{\circ} 72,2020$; MORENO I GENÉ, Josep, "El carácter laboral de la prestación de servicios de reparto a través de la plataforma digital Glovo el Tribunal Supremo zanja el debate", Iuslabor, no 3, 2020; Todolí SIGNES, Adrián "Comentario a la Sentencia del Tribunal Supremo español que considera a los Riders empleados laborales.", Labour Law \& Issues, vol. 6, $\mathrm{n}^{\circ}$ 2, 2020, disponible en https://adriantodoli.com/2020/09/30/comentario-a-la-sentencia-deltribunal-supremo-sobre-los-falsos-autonomos-en-glovo/; Sobre el tema, con anterioridad a la Sentencia particularmente interesante sobre la aplicación de los indicios de laboralidad al trabajo en plataformas ver, Goerlich Peset, Jose Maria, García Rubio, María Amparo, "Indicios de autonomía y de laboralidad en los servicios de los trabajadores en plataforma”, en Coord., Pérez De Los CoBos OriHUEL, Francisco, El trabajo en plataformas digitales, Wolters Kluwer, 2018, NiETO ROJAS, Patricia, "Nuevamente sobre la laboralidad del vínculo contractual de los prestadores de servicios en plataformas digitales", Iuslabor, $\mathrm{n}^{\circ} 1$, 2019; Suárez Corujo, Borja, "La gran transición: la economía de plataformas digitales y su proyección en el ámbito laboral y de la seguridad social", Temas Laborales, no 141, 2018, p. 37-66; TRILLO PÁRRAGA, Francisco, "Calificación jurídica del trabajo en plataformas digitales. A propósito de la SJSOC 128/19, de 3 de abril", Nueva revista española de derecho del trabajo, n 224, 2019, p. 257-266; GORELLI HERNÁNDEZ, Juan, "Paso a paso hacia el Tribunal Supremo el problema de la laboralidad de la prestación de servicios en plataformas digitales (casos Glovo y Deliveroo)", $T y D$, n ${ }^{\circ}$ 60, 2019, p. 75-88; MORENO I GENÉ, Josep "El carácter laboral de la prestación de servicios a través de plataformas digitales. el caso de Glovo. Comentario de la Sentencia del Tribunal Superior de Justicia de Cataluña de 21 de febrero de 2020", Revista General de Derecho del Trabajo y de la Seguridad Social, n 57, 2020; PAZOS PÉrEZ, Alexandre, "Sobre 
De esta forma, habiendo ya resuelto el Tribunal Supremo que materialmente un repartidor de plataformas digitales es un asalariado, la nueva normativa pretende incorporar los elementos de juicio usados por el Tribunal, para que tengan efectos generales (sobre todos los repartidores y sobre todas las compañías de plataformas dedicadas al reparto).

\subsection{Contenido de la nueva presunción}

\subsubsection{Presunción e inversión de la carga de la prueba}

La nueva normativa no modifica el concepto de trabajador, esto es, no tiene efectos materiales, sino solamente de carga de la prueba. De esta forma, el ámbito subjetivo de aplicación del Estatuto de los trabajadores sigue determinado por su artículo primero. Tanto es así, que la nueva DA23 se encarga de recordar expresamente que esta "nueva" presunción, no modifica la exclusión realizada por el artículo 1.3. ET. Esto significa que, a pesar de la "nueva" presunción específica sobre la laboralidad de los repartidores, los trasportistas que ostenten la titularidad de las autorizaciones administrativas necesarias para realizar la actividad seguirán excluidos de la laboralidad por mor del artículo 1.3 ET. Esta adición final en el RDL 9/2021 no parece que fuera realmente necesaria ${ }^{20}$, dado que las presunciones, por definición, no modifican el derecho sustantivo. De esta forma, quién es trabajador laboral y quién no, no parece que quede modificado por la DA23, limitándose a establecer la carga de la prueba de la realidad material de la relación en los hombros de quién sostenga que la relación no es de carácter laboral (algo que ya existía anteriormente derivado del artículo 8.1 ET).

En cualquier caso, el "recordatorio" de que la nueva DA 23 no afecta a las exclusiones determinadas en el artículo 1.3 ET, sirve para despejar cualquier duda respecto del carácter meramente procesal (carga de la prueba) de esta normativa.

El Tribunal Supremo, ya reconocía, vía artículo 8.1 ET, la existencia de una presunción de laboralidad con anterioridad a esta regulación, por tanto, esta nueva DA23 solamente clarifica, o más bien específica, que dicha presunción es también aplicable a los casos en los que la prestación de servicios se realiza en materia de plataformas digitales usando un algoritmo para la gestión de los trabajadores. Una aclaración que, aunque probablemente nada aporte sustantivamente, sí puede tener relevancia a efectos prácticos con objeto de reducir la alta conflictividad -número de juicios- y dispersión judicial -sentencias contradictorias- que se estaban produciendo. El hecho de que las empresas modifiquen

el empleo en plataformas digitales. Comentario de la sentencia del Juzgado de lo Social núm. 1 de Madrid, de 4 de abril de 2019", Nueva revista española de derecho del trabajo, $\mathrm{n}^{\circ} 224,2019$, p. 247-256.

${ }^{20}$ RodríGUeZ-PIÑERo Royo, Miguel, "Por fin, la ley Rider", en Trabajo, persona, Derecho y mercado, 2021, disponible en http://grupo.us.es/iwpr/2021/05/13/por-fin-la-ley-rider/. 
repetidamente su relación contractual y su forma de funcionar con los repartidores con objeto de evitar la aplicación de la normativa laboral, junto con el hecho de que las diferentes sentencias incluidas del Tribunal Supremo no hayan conseguido la laboralización de los repartidores de plataformas, puede justificar la necesidad de una norma que ponga fin a esta situación de permanente "huida del Derecho del trabajo".

Una cuestión que merece especial atención es la técnica legislativa usada -sin olvidar que es la decidida por los agentes sociales-. En efecto, se está ante una presunción de laboralidad para cuya activación se establecen más requisitos que para activar la presunción general del artículo 8.1 ET. Así, el artículo 8.1 ET, de acuerdo con la jurisprudencia, implica que la inversión de la carga de la prueba se producirá siempre que exista un contrato de prestación de servicios retribuido. Esto viene a significar que la carga de la prueba del trabajador, en un caso de falsos autónomos, se limita a demostrar que presta servicios a cambio de una retribución para el empresario. Una vez acreditados estos extremos, el juez declarará la laboralidad salvo que el empresario sea capaz de demostrar que dicha prestación de servicios se realiza con independencia y por cuenta propia del prestador de servicios. Sin embargo, la presunción establecida por la DA23 exige -o parece exigir- bastantes más requisitos para su aplicación: i) que las actividades sean de reparto o distribución; ii) que la empleadora ejerza facultades de dirección de forma directa o implícita a través de una plataforma digital; iii) que se use un algoritmo para gestionar el servicio o para determinar las condiciones de trabajo.

De esta forma, el ámbito de aplicación de la presunción general (artículo 8.1 ET), al menos en apariencia, se ve incluso reducido. No obstante, tendría poco sentido interpretar que una normativa, que tiene por objetivo reducir la conflictividad y conseguir la laboralidad de los repartidores en plataformas, añada una carga superior de presupuestos o requisitos de "activación" de los ya existentes antes de la norma.

La razón para esta concreta redacción no parece que sea otra que las dificultades de alcanzar un acuerdo en el diálogo social. De esta forma, esta norma es el producto de una composición de intereses contrapuestos. De un lado, tenemos una formulación en forma de "presunción", deseada por la parte patronal, que reduce la efectividad de la norma al limitar sus efectos a los de carácter procesal. Por el otro lado, se está ante una formulación de inclusión declarativa, deseada por los sindicatos, que implica que todos aquellos que cumplan una serie de requisitos serán "automáticamente" incluidos en el Estatuto de los trabajadores. Así, la norma final publicada en el BOE acaba incorporando las dos facetas: de un lado, se establece en forma de presunción, de otro lado, se fija una serie de requisitos que, en caso de cumplirse, implicarán que se está materialmente, y no solo procesalmente, ante un trabajador laboral. 
En fin, parece que existen dos formas de interpretar este artículo: bien entender que se está ante un presunción iuris tantum ${ }^{21}$; bien entender que se está ante una presunción iuris et de iure $^{22}$ si se cumplen los requisitos de la DA23, esto es, i) que las actividades de reparto o distribución sean personales y retribuidas; ii) que la empleadora ejerza facultades de dirección de forma directa, indirecta o implícita a través de una plataforma digital; iii) que se use un algoritmo para gestionar el servicio o para determinar las condiciones de trabajo.

Entre estas dos opciones, en mi opinión, se debe decantar por la primera. Primero, derivado de la referencia al artículo 8.1 ET que conduce a una presunción iuris tantum (que admite prueba en contrario). En segundo lugar, porque la propia DA23 establece que, dentro de su redacción, que "Esta presunción no afecta a lo previsto en el artículo 1.3 de la presente norma". De esta forma, parece que, incluso aunque se consiga acreditar todos requisitos exigidos por la nueva presunción de la DA 23 ET, el juez deberá rechazar la laboralidad si la empresa consigue demostrar que se está ante una de las exclusiones del 1.3 ET (lo que parece implicar que la DA23 admite prueba en contra).

No obstante, la solución judicial probablemente pase por una posición intermedia, esto es, cuando se den los requisitos establecidos en la DA23 las posibilidades judiciales de exclusión se verán seriamente reducidas ${ }^{23}$. En efecto, la parte "declarativa" de la norma indica que, si se dan los tres requisitos de la DA 23 ET, se deberá entender que existe dependencia y ajenidad teniendo que justificar la exclusión de la laboralidad en algún otro criterio distinto a la dependencia o la ajenidad (ejemplo, exclusión declarativa de los trasportistas con autorización administrativa).

\section{a) ¿Subcontratación?}

Tras la aprobación de esta DA 23 ET, una de las primeras cuestiones que se plantea es la validez de la subcontratación del servicio de reparto por parte de las empresas de plataformas. Esto es, ¿esta nueva DA23 ET afecta a la licitud o al régimen jurídico de la subcontratación?

\footnotetext{
${ }^{21}$ Tesis defendida por, RodríGUEZ-PIÑERo Royo, Miguel, "Por fin, la ley Rider", en Trabajo, persona, Derecho y mercado, 2021; SANGUINETTI, Wilfredo, "La presunción..." op cit.

${ }^{22}$ Así, parte de la doctrina defiende que se está ante una presunción "plena" que si bien no tiene porqué encajar completamente con el contenido jurídico de una presunción iuris et de iure viene a significar la inclusión de los riders en el ámbito de aplicación de las normas laborales si se cumplen esos requisitos, CRUZ Villalón, Jesús, op cit., BAylos Grau, Antonio op cit., y Rojo ToRRECILlas, Eduardo op cit.

${ }^{23}$ RodríGUeZ-PIÑERo Royo, Miguel, "Por fin, la ley Rider", en Trabajo, persona, Derecho y mercado, 2021, disponible en http://grupo.us.es/iwpr/2021/05/13/por-fin-la-ley-rider/.
} 
La respuesta inicial debe ser negativa. La DA 23 ET tiene como objetivo que la persona que materialmente realiza las funciones de reparto esté contratada laboralmente, pero nada parece obligar a que esta contratación la realice directamente la empresa propietaria de la plataforma. Así, la licitud de la subcontratación por parte de la plataforma de terceras empresas será determinada por las reglas comunes a la subcontratación (artículo 42 y 43 ET), sin que nada nuevo parezca incluir este artículo. En este sentido, cabe traer a colación el acta de infracción de la ITSS de Barcelona en materia de cesión ilegal entre Cabify y la empresa de VTC que contrata a los conductores que prestan servicios para Cabify ${ }^{24}$. En este supuesto la Inspección de Trabajo consideró que la subcontratación por parte de Cabify a una empresa externa no es lícita dado que esa tercera empresa no dispone de la estructura productiva esencial para realizar la prestación de servicios -la plataforma pertenece a la principal- $\mathrm{y}$, adicionalmente, el poder directivo sobre los trabajadores es ejercido directamente por el algoritmo del que es propietario la plataforma. Esto es, el poder de dirección sobre los trabajadores no es ejercido realmente por la empresa que contrata a los trabajadores, sino por la empresa principal.

En cualquier caso, como aquí se señala, la licitud o no de la subcontratación en las plataformas no dependerá de la nueva DA23 ET, sino de los criterios clásicos en materia de cesión ilegal de trabajadores. Eso sí, lo que sí determina esta nueva disposición es que esa empresa subcontratada por la plataforma tenga como asalariado al trabajador que materialmente realiza los repartos (salvo que sea de aplicación alguna exclusión del 1.3 ET).

b) ¿Socios cooperativistas?

Otra cuestión que se plantea es si la DA23 ET excluye la posibilidad de realizar tareas de reparto mediante plataformas a través de repartidores calificados como socios cooperativistas -y, por tanto, excluidos del Estatuto de los trabajadores-. En este sentido, en apariencia nos encontramos ante dos normativas contradictorias: de un lado, la normativa sobre cooperativas que permite a las mismas disponer de trabajadores socios cooperativistas en régimen de autonomía y, por tanto, excluido del ámbito de aplicación del Estatuto de los trabajadores; y de otro lado, la DA23 ET, que incluye en el ámbito de aplicación del Estatuto los repartidores a través de plataformas digitales. Así, ¿cuál de las dos normas aplicaríamos a una cooperativa de reparto a través de plataformas digitales?

\footnotetext{
24 Todolí Signes, Adrián, “Argumentos de la sanción a Cabify por cesión ilegal de la Inspección de trabajo -aplicables a muchas de las plataformas digitales-", Argumentos en Derecho laboral, 2021, disponible en https://adriantodoli.com/2021/03/25/argumentos-de-la-sancion-a-cabify-por-cesion-ilegal-de-lainspeccion-de-trabajo-aplicables-a-muchas-de-las-plataformas-digitales/
} 
El principio de especialización, que suele servir para decidir qué norma aplicar (la norma especial prima sobre la norma general) no parece que será de mucha utilidad, dado que ambas normas son especiales: una por la especialidad de la cooperativa, la otra por la especialidad del sector del reparto en plataformas digitales.

La solución a esta aparente contradicción parece estar en la propia DA 23 ET. En efecto, esta norma aclara que no afectará a las exclusiones del artículo 1.3 ET. Entre estas exclusiones, si bien no se encuentra directamente la exclusión de los socios cooperativistas, sí se establece una exclusión general en los siguientes términos: $1.3 \mathrm{~g}$ ) ET "todo trabajo que se efectúe en desarrollo de relación distinta de la que define el apartado 1". De esta manera, la propia configuración de la nueva normativa (DA 23 ET), en el fondo, la hace ceder ante cualquier otra norma que elimine la laboralidad.

\subsubsection{Reducción de intensidad del elemento de dependencia jurídica}

Uno de los elementos de carácter sustantivo más novedosos de la DA23 ET consiste en la reducción de intensidad del poder de dirección -dependencia jurídica- como elemento necesario para la calificación como laboral de un trabajador ${ }^{25}$. En España, cinco son las notas características del contrato de trabajo. No obstante, de estas cinco dos son las que permiten diferenciar el contrato de trabajo de otras figures afines en materia de prestación de servicios: la dependencia jurídica y la ajenidad. En este contexto, el Tribunal Supremo, fundamentándose en la necesidad de adaptar la interpretación del concepto de trabajador a las nuevas realidades económicas y organizativas de las empresas actualmente (artículo 3 Código Civil), ha venido en las últimas décadas reduciendo el protagonismo de la dependencia jurídica como criterio determinante. Esto es, en sucesivas sentencias, el Tribunal Supremo, ha admitido la relación laboral a pesar de que existiera un ejercicio

\footnotetext{
${ }^{25}$ Sobre los elementos del concepto de trabajador se puede ver, BAYÓN CHACÓN, Gaspar, PÉREZ BOTIJA, Eugenio, Manual de Derecho del Trabajo, Marcial Pons, Madrid, 1976 p. 16; y en DE LA VILLA GIL, Luis Enrique, El trabajo a domicilio, Aranzadi, Madrid, 1966, p. 135. Por otra parte, Alonso OlEA, Manuel, Introducción al Derecho del Trabajo, ed. Revista de Derecho Privado, Madrid, 1968, p. 18 y 19, se decanta por defender la "ajenidad en los frutos" como elemento que distingue a la relación laboral de otros contratos civiles. En un sentido parecido, pero con la ventaja de poder ser aplicado también a prestaciones de servicio -y no solamente a trabajo industrial-, MONTOYA MELGAR, Alfredo, "El poder de dirección del empresario", IEP, Madrid, 1965, p. 72, se decanta por la "ajenidad en la utilidad patrimonial" No obstante este autor reconoce, matizadamente, también la "dependencia" como característica clasificatoria del contrato de trabajo. Finalmente, ALBIOL MoNTESINOS Ignacio., "Entorno a la polémica ajenidad-dependencia", CCDT, $\mathrm{n}^{\circ}$ 1, 1971, p. 41, describe la "ajenidad en los medios de producción” y ya, posteriormente ALARCÓN CARACUEL, Manuel Ramon., introduce la ajenidad en el mercado en "La ajenidad en el mercado: Un criterio definitorio del contrato de trabajo" Civitas, no 28, 1986, SALA FrAnCO, Tomás y LóPEz MorA, Federico, "Contrato de trabajo", Comentarios a las leyes laborales. El Estatuto de los Trabajadores, Tomo I, 1990; PÉreZ DE Los Cobos ORIHUEL, Francisco, "El trabajo subordinado como tipo contractual", Documentación Laboral, no 39, 1993.
} 
casi inexistente del poder de dirección del empresario sobre el prestador de servicios, si se daban otros elementos ${ }^{26}$. El Tribunal Supremo ha realizado esta interpretación flexible en multitud de sectores cualificados y no cualificados: limpiadores, médicos, arquitectos, profesores de academia, traductores, dobladores, repartidores, etc. Sin embargo, con esta DA 23 ET se eleva a rango normativo, por primera vez, esta menor exigencia de dependencia jurídica dentro del contrato de trabajo, al menos, para el sector de los repartidores.

En la práctica, en materia de plataformas digitales, la cuestión de la existencia o inexistencia del poder de dirección de la plataforma sobre el trabajador es el elemento más discutido en la jurisprudencia. Así, las plataformas alegan que el trabajador dispone de absoluta libertad para elegir su horario, para elegir dónde trabajar, durante cuánto tiempo e incluso para rechazar tareas asignadas sin que haya consecuencias negativas. Con este argumento, se pretendió demostrar que el requisito de dependencia jurídica exigido por la normativa no se daba, lo que debía conducir a la exclusión de la laboralidad de estos trabajadores.

Dado que este es el argumento central de las plataformas, la nueva norma le dedica especial atención. Así, la regulación, viene a señalar que se considerará cumplido el requisito de dependencia jurídica siempre que las facultades de organización, dirección y control del empresario se ejerzan a través de un algoritmo. Además, señala que ese ejercicio de las facultades podrá ser directo, indirecto o implícito. De esta forma, siempre que el algoritmo determine u oriente las condiciones de la prestación del servicio o las condiciones de trabajo se entenderá que se cumple el requisito de dependencia jurídica necesario para la laboralidad.

Consecuentemente, algunos ejemplos que conducirían a la inclusión del repartidor en el Estatuto de los trabajadores podrían ser que el algoritmo asigne las tareas a realizar jornada- aun con posibilidad de rechazo por parte del repartidor, determine quién realiza cada tarea -jornada-, se establezcan incentivos (ejemplo, mayor salario cuando llueve o en horario de fin de semana), defina cómo se evalúa el trabajo (ejemplo, reputación digital $)^{27}$-control-, resuelva cuándo alguien es "desactivado" de la aplicación -poder disciplinario-, etc.

\footnotetext{
${ }^{26}$ TODOLÍ SIGNES, Adrián, "Plataformas digitales y concepto de trabajador: una propuesta de interpretación finalista", Lan harremanak, $\mathrm{n}^{\circ}$ 41, 2019.

${ }^{27}$ Sobre la reputación digital ver, TODOLí SIGNES, Adrián, "La evaluación de los trabajadores por parte de los clientes como método de vigilancia y control en la empresa: reputación online y protección de datos", CEF.RTSS, nº 427, 2018, p. 63-90.
} 
Téngase en cuenta que, aunque las condiciones del algoritmo respondan a cuestiones "objetivas" o "lógicas", esto no excluirá la laboralidad. Así, que la empresa precise que el repartidor más cercano al lugar de recogida es al que se le asignará la tarea, no deja de ser una decisión empresarial que tiene como resultado una manifestación del poder de dirección a través de unas instrucciones escritas en el algoritmo.

Adicionalmente, la normativa establece que este ejercicio del poder empresarial se puede realizar de forma indirecta o implícita. Esto hace referencia específicamente al establecimiento de opciones entre las que el trabajador puede elegir. De hecho, es común que la empresa no concrete las condiciones exactas de la prestación de servicios del trabajador, sino que permita un abanico de opciones entre los que el repartidor puede elegir (ej, elegir horario). En este sentido, la normativa parece indicar que, aunque finalmente el repartidor pueda elegir entre una serie de opciones disponibles en la plataforma, el hecho de que las opciones hayan sido presentadas y determinadas conforme a los deseos de la empresa dueña de la plataforma será suficiente para entender que se está ante un ejercicio (indirecto o implícito) del poder de dirección.

En el mismo sentido, se debe entender que, si el algoritmo fija una serie de opciones para que el consumidor final elija, implicando consecuencias para el trabajador, también se cumplirá el requisito de dependencia jurídica. Una vez más, aunque sea el consumidor final el que realice la elección, lo hará dentro de un sistema decidido por el empresario, dueño de la plataforma, lo que implicará que está realizando un ejercicio indirecto o implícito de su poder de dirección siempre que acabe repercutiendo de alguna forma en el trabajador. Así, por ejemplo, aunque el consumidor final pudiera elegir el repartidor entre una lista de "posibles" repartidores, seguiría siendo el algoritmo el que ordena los repartidores sesgando con ello la decisión del consumidor final.

A mayor abundamiento, la decisión tomada por el consumidor final se basará en los datos o información que la plataforma decida mostrarle, interviniendo de nuevo, aunque sea de forma indirecta, en el resultado de esa elección y las consecuencias que la misma tiene para el trabajador. Pero no solo eso, sino que se debe recordar que, en cualquier caso, la decisión de permitir al consumidor final elegir al repartidor concreto sigue siendo una opción empresarial por la que manifiesta su poder de dirección.

En definitiva, la nueva legislación rebaja sumamente el requisito de dependencia jurídica, aceptando que esta se produce mediante una diversidad de actuaciones empresariales entre las que debe incluirse usar algoritmos que presenten una serie de opciones entre las que el repartidor, o cliente final, puede elegir, siempre que las mismas afecten a la prestación de servicios o a sus condiciones laborales. 


\subsection{Trascendencia de la nueva presunción}

Como se ha señalado, el artículo 8.1 ET ya incluía una presunción iuris tantum de laboralidad, así pues, la trascendencia jurídico-laboral de la nueva DA 23 ET se limita, en mi opinión, a dos cuestiones: de un lado, la reducción de la intensidad en el requisito de dependencia jurídica; de otro lado, que es el resultado de un acuerdo en el marco del diálogo social.

1) En primer lugar, como se ha visto, esta norma eleva a rango normativo una interpretación judicial, por la cual la gestión algorítmica del trabajo se debe considerar ejercicio del poder de dirección y control a efectos de la calificación jurídico-laboral como trabajador. Si bien es cierto que, dado el limitado ámbito de aplicación (plataformas digitales del sector del reparto), sus efectos serán escasos, no se debe descartar que esta regulación sirva, vía interpretativa, para establecer los estándares en materia de ámbito subjetivo de aplicación del Estatuto de los trabajadores en el resto de sectores del trabajo en plataformas.

En este sentido, no debería olvidarse que la DA 23 ET eleva a rango de ley una interpretación judicial del Tribunal Supremo que viene siendo aplicada en las últimas décadas a otros sectores. Así pues, esta DA 23 ET se convierte en una "aprobación" por parte del legislador de la línea interpretativa tomada por el Tribunal Supremo. De esta forma, esto puede dar "alas" al Tribunal Supremo en su reinterpretación del concepto de trabajador a efectos de reducir o eliminar el requisito de dependencia jurídica para la calificación del contrato de trabajo en otros sectores productivos conforme a las nuevas realidades organizativo-empresariales. Algo que, aunque actualmente ya venía haciendo, ahora lo podrá realizar con una mayor legitimidad ${ }^{28}$.

2) En segundo lugar, con independencia del escaso contenido jurídico-obligacional de la nueva norma, su trascendencia puede provenir de la forma en que se ha realizado ${ }^{29}$. En efecto, esta normativa es el resultado de un acuerdo en el marco del Diálogo social tripartito a nivel Estatal. Un acuerdo que no solo implica, en principio, una voluntad de

\footnotetext{
${ }^{28}$ Como bien señala ROJO TORRECILLAS, Eduardo, "Y llegó la norma que declara la relación laboral de "las personas dedicadas al reparto en el ámbito de las plataformas digitales. Primeras notas y comentarios al RDL 9/2021 de 11 de mayo", El nuevo y cambiante mundo del trabajo. una mirada abierta y crítica a las nuevas realidades laborales, 2021.

${ }^{29}$ Tesis también apoyada por gran parte de la doctrina en sus primeras valoraciones ver, CRUZ VILLALÓN, Jesus., "Una presunción plena de laboralidad de los riders", Blog de Cruz Villalón, 2021, PÉREZ DEL PRADO, Daniel., "La Ley Rider: un muy buen comienzo", Foro de labos, 2021; SANGUinetTI, Wilfredo, "La presunción de laboralidad de los riders o cómo se consiguió la cuadratura del círculo de la laboralidad", $E l$ blog de Wilfredo Sanguinetti, 2021, BAYLOS GRAU, Antonio, "Por fin la norma sobre los repartidores de plataformas (la ley “riders")", según Antonio Baylos, 2021.
} 
cumplimiento en su contenido, sino también la aquiescencia en la necesidad de una regulación que ponga fin al debate judicial. Poca duda cabe que, el hecho de que exista un acuerdo entre la patronal y los sindicatos más representativos, le da un plus de legitimidad a la normativa en favor de todos los operadores jurídicos que deban hacerla cumplir.

También ello abre el camino para la aplicación de la misma solución jurídica en otros sectores donde se produce un uso masivo de los falsos autónomos. Así, eliminado el efecto social y mediático de los "rider", la realidad es que jurídicamente existen pocas razones para no aplicar similar desenlace (la laboralidad) en otros sectores de plataformas -y no de plataformas- cuyo uso abusivo en las últimas décadas de la figura del autónomo ha desfigurado la aplicación del Derecho del trabajo. De esta forma, bien legalmente, bien judicialmente, esta normativa puede implicar un primer paso para tomar medidas que encaucen esta actual disfuncionalidad.

\section{El deber de trasparencia algorítmica}

\subsection{Derecho de información de los representantes de los trabajadores sobre los algoritmos}

El impacto social y mediático de las plataformas digitales no se ha limitado al debate sobre el concepto de trabajador, sino que ha puesto de relieve el algoritmo como herramienta de gestión empresarial con efectos sobre los trabajadores más allá del mundo de las plataformas. Conforme la doctrina viene señalando en los últimos años ${ }^{30}$, cada vez

\footnotetext{
${ }^{30}$ DEMBE, Aron., et al., , "The impact of overtime and long work hours on occupational injuries and illnesses: new evidence from the United States", Occupational and Environmental Medicine, vol. 62, n 9, 2005, p. 588-597; DOMEINSKI, Juliane, et al., "Human redundancy in automation monitoring: Effects of social loafing and social compensation", en Proceeding of human factors and ergonomics Society 51st Annual meeting, 2007, p. 587-591; HuNG, Walter, et al., "Managing the risk of overusing mobile phones in the working environment: a study of ubiquitous techno-stress", PACIS 2011 Proceedings, Paper 81, 2011; SCHUMACHER, Susan, "What employees should know about electronic performance monitoring" ESSAI 8, n 28, 2011, p. 138-144; EU-OSHA, Monitoring technology: the 21st century's pursuit of wellbeing?, 2017 disponible en https://osha.europa.eu/en/tools-and-publications/publications/monitoringtechnology-workplace/view; EU-OSHA, Foresight on new and emerging occupational safety and health risks associated with digitalisation by 2025, 2018 disponible en https://osha.europa.eu/en/tools-andpublications/publications/foresight-new-and-emerging-occupational-safety-and-health-risks/view

DERKS, Daantje, and BAKKER, Arnold B, "Smartphone use, work-home interference and burnout: a diary study on the role of recovery", Applied Psychology, nº 63 (3), 2014; PÉreZ ZAPATA, Óscar, et al., "Digitalización, intensificación del trabajo y salud de los trabajadores españoles", 2019, (disponible en https://www.ccoo.es/24c0e370fa4b4d1f3682b1780854af9c000001.pdf. ; PÉREZ ZAPATA, Óscar., Trabajo sin límites, salud insostenible: La intensificación del trabajo del conocimiento (e-prints, Universidad Complutense de Madrid), 2015; LINDSAY, Greg, "We spent two weeks wearing employee Trackers: Here's
} 
es más habitual que las empresas usen los algoritmos o la inteligencia artificial para tomar decisiones que afectan a los trabajadores (contrataciones y despidos, determinación de cuadrantes de horarios, evaluación de los trabajadores, ascensos, etc.). Unos métodos que muchas veces son desconocidos para los propios trabajadores que consideran que dichas decisiones son tomadas por humanos cuando realmente vienen apoyadas por esta tecnología o directamente el responsable humano se limita a comunicar lo que el algoritmo ha decidido.

Cabe decir que, actualmente, el RGPD ya regulaba la obligación de la empresa de informar al trabajador cuando estos métodos de procesamiento automatizado y de creación de perfiles eran usados. Un derecho de alcance individual que, en muchas ocasiones, se incumplía dada la incapacidad del trabajador individual, de un lado, de conocer cuando se estaba incumpliendo esta obligación, de otro lado, por el propio miedo del trabajador a represalias si decidía reclamarlo. Por esta razón, la doctrina ha criticado al RGPD por olvidar la faceta colectiva y sindical del mundo del trabajo y no haber concedido derechos de información y negociación sobre el algoritmo a los representantes de los trabajadores ${ }^{31}$.

what we learned", Fact Coexist, 2015, https://www.fastcompany.com/3051324/we-spent-two-weekswearing-employee-trackers-heres-what-we-learned; AKHTAR, Jam, and MOORE, Phoebe, "The PsychoSocial Impacts of Technological Change in Contemporary Workplaces and Trade Union Responses" International Journal of Labor Research, vol. 8, $\mathrm{n}^{\circ}$ 1-2, 2016, p. 102-131; AJUNwA, Ifeoma, et al., "Limitless worker surveillance", California Law Review, vol. 105, n 3, 2017; HORTON, John, et al., "Workplace Safety Futures: The impact of emerging technologies and platforms on work health and safety and workers' compensation over the next 20 years", CSIRO, Canberra, 2018; FERNÁNDEZ AVILÉS, José Antonio, "NTIC y riesgos psicosociales en el trabajo: estado de situación y propuestas de mejora", $D S L, \mathrm{n}^{\circ}$ 2, 2017; RODRÍGUEZ-RICO, Roldan, "Los retos para la prevención de riesgos laborales ante la tecnificación del trabajo", in Cerejeria, Namora; Nuno, et al., Health at work, ageing and environmental effects on future social security and labour law Systems. Cambridge Scholars Publishing, 2018; MOORE, Phoebe Work and the GDPR: The future for algorithms and people analytics Available at https://staffblogs.le.ac.uk/management/2018/09/20/work-and-the-gdpr-the-future-for-algorithms-andpeople-analytics/ 2017; MOORE, Phoebe, The quantified self in precarity. Work, technology and what counts, New York, Routledge, 2018; MOORE, Phoebe, Humans and machines at work: monitoring, surveillance and automation in contemporary capitalism, London. Palgrave Macmillan, 2018; MOORE, Phoebe, Data subjects, digital surveillance, AI and the future of work, European Parliament Science and Technology Office, Brussels, 2020; UTS, Heat Stress and On-Demand work: The experience of food delivery and courier cyclists. Climate Justice Centre, 2019, disponible en https://opus.lib.uts.edu.au/bitstream/10453/134736/1/On\%20Demand\%20cyclists\%20UTS\%20final.pdf; TODOLí SigNes, Adrián, "En cumplimiento de la primera Ley de la robótica: Análisis de los riesgos laborales asociados a un algoritmo artificial / inteligencia dirigiendo el trabajo", Labor Law \& Issues, vol. 5, $\mathrm{n}^{\mathrm{o}} 2$ 2, 2019, disponible en https://papers.ssrn.com/sol3/papers.cfm?abstract_id=3506584.

${ }^{31}$ TODOLí SigNES, Adrián, "La gobernanza colectiva de la protección de datos en las relaciones laborales: big data, creación de perfiles, decisiones empresariales automatizadas y los derechos colectivos", $R D S, \mathrm{n}^{\circ}$ 84, 2018, p. 69-88; Molina Navarrete, Cristóbal, Datos y derechos digitales de las personas trabajadoras en tiempos de (pos)covid19: entre eficiencia de gestión y garantías, Bomarzo, 2021. 
Un "defecto" que el RDL 9/2021 pretende solventar con la introducción de una nueva letra d) en el apartado 4 del artículo 64 (relativo a los derechos de información y consulta del comité de empresa), que tendrá la siguiente redacción:

d) Ser informado por la empresa de los parámetros, reglas e instrucciones en los que se basan los algoritmos o sistemas de inteligencia artificial que afectan a la toma de decisiones que pueden incidir en las condiciones de trabajo, el acceso y mantenimiento del empleo, incluida la elaboración de perfiles.

Así, el comité de empresa tendrá derecho a conocer, no solamente si existe algún algoritmo que tome decisiones o influya en las condiciones de trabajo, acceso o mantenimiento del empleo, sino también a saber los parámetros, reglas e instrucciones de funcionamiento de dicho algoritmo o sistema de inteligencia artificial. Es importante señalar que esta obligación se incluye para todo tipo de empresas (sean de plataformas o no) y para todo tipo de sectores ${ }^{32}$. De esta forma, aunque la norma que aquí se comenta se ha bautizado como "ley rider", la realidad es que la mitad de sus preceptos (uno de dos) es una modificación que afecta a todo tipo de empresas, en todos los sectores.

\subsection{Concepto de algoritmo}

La introducción en el Estatuto de los trabajadores de una regulación sobre los algoritmos plantea una primera pregunta ¿qué es un algoritmo?

En este sentido, la RAE define algoritmo como el "Conjunto ordenado y finito de operaciones que permite hallar la solución de un problema". De esta forma, lo primero que se debe hacer es desligar el concepto de algoritmo del de programa informático (software). En efecto, un algoritmo no es más que un procedimiento metodológico que permite solucionar un problema, sin que sea necesario que para ello se use un programa informático -aunque habitualmente se haga para agilizar y simplificar la aplicación del procedimiento o de las operaciones necesarias. En este sentido, los representantes de los trabajadores tendrán derecho a conocer cualquier sistema (conjunto ordenado de operaciones) usado por la empresa con objeto de incidir en las condiciones de trabajo, el acceso y mantenimiento del empleo, incluida la elaboración de perfiles.

El uso del término "algoritmo", debe ponerse en contexto con la normativa europea, a pesar de que la terminología no encaje perfectamente. En la regulación europea se usa un concepto más restrictivo que el "algoritmo". Así, el RGPD enmarca los derechos individuales frente a la inteligencia artificial y los algoritmos mediante el concepto de

\footnotetext{
${ }^{32}$ Molina NAVARRETE, Cristóbal, "La "Ley" de personas repartidoras en plataformas online ("riders"):
} ¿pequeño paso legal, gran paso para humanizar el precariado digital?”, Transformaework, 2021. 
"decisiones automatizadas". En este sentido, en la regulación europea, para aplicar la normativa especial, la empresa debió establecer un conjunto de reglas que, de forma autónoma y automática -sin intervención humana-, llegan a una solución (ej, decidir a qué trabajador se contrata de entre distintos candidatos o decidir qué candidatos se descartan del proceso de selección).

Así, aunque el nuevo apartado d) del artículo 64. ET no incluya expresamente ese requisito de "automaticidad" exigido en Europa, la realidad es que todo algoritmo, para ser considerado tal, debe permitir hallar una solución -una decisión o una respuesta-, sin intervención humana. En este sentido, el conjunto ordenado y finito de operaciones debe funcionar de forma automática -una operación tras otra- hasta alcanzar la solución buscada en su programación.

\subsection{Comparativa entre los nuevos derechos de información y la regulación europea ya existente}

La principal diferencia entre los derechos de información del art 64 ET en materia de algoritmos y la regulación del artículo 22 RGPD, como ya se ha indicado, es que los primeros son de carácter colectivo y los segundos de carácter individual. Sin embargo, aquí no terminan las diferencias.

En este sentido, el RDL 9/2021 otorga derechos de información en favor de los representantes de los trabajadores en muchos más supuestos que lo hace el artículo 22 RGPD, pero con efectos menores.

Así, para la activación del artículo 22 RGPD se requiere que las decisiones automáticas se tomen sin intervención humana significativa ${ }^{33}$. Por el contrario, letra d) en el apartado 4 del artículo 64, solamente exige que dichos algoritmos afecten a la toma de decisiones que puedan incidir sobre el trabajador. Así, aunque se use el algoritmo como simple apoyo a la toma de decisiones por parte del empresario se aplicará el artículo 64.4 d) ET. Dicho en otras palabras, aunque el algoritmo no sea determinante para la decisión final tomada sobre el trabajador, su mero uso, implicará el nacimiento de los derechos de información de los representantes de los trabajadores. Resultando, en definitiva, que la aplicación del artículo 64.4 d) ET se dará en muchos más casos que el artículo 22 RGPD.

En la otra cara de la moneda, los efectos del artículo 22 RGPD son mucho mayores, cuando efectivamente es de aplicación, que los meros derechos de información de carácter colectivo del artículo 64.4 d) ET. Así, el artículo 22 RGPD establece, como primera

\footnotetext{
${ }^{33}$ Como establece el GT29 en Guidelines on Automated individual decisión-making and Profiling for the purposes of Regulation 2016/679, Adoptadas el 3 de octubre de 2017, p. 21.
} 
garantía existente en caso de decisiones basadas únicamente en el tratamiento automatizado de datos, su prohibición. En este sentido, el artículo 22 RGPD concede el derecho a no ser objeto de este tipo de decisiones, derecho que el GT29 interpreta como una prohibición -sin necesidad de reclamar activamente el derecho- a los responsables de datos de tomar las decisiones con esta metodología automatizada ${ }^{34}$. No obstante, esta es una prohibición relativa dado que existen excepciones, a saber: a) que sea necesaria para la celebración o la ejecución de un contrato; b) que esté autorizada por el Derecho interno de un Estado Miembro; c) que se cuente con el consentimiento explícito del interesado. Ahora bien, dado que, en el contrato de trabajo, el consentimiento del trabajador, por regla general, no será válido ${ }^{35}$-y a falta de una regulación nacional-, solamente se podrá entender válida la decisión automatizada si es necesaria para la celebración o ejecución del contrato de trabajo (excepción (a)).

A su vez, en caso de que una de las excepciones a la prohibición de uso de los algoritmos fuera aplicable (necesidad para cumplir con el contrato) el RGPD, en el artículo 22.3, establece una serie de garantías que la empresa debe cumplir "para salvaguarda de los derechos y libertades y los intereses legítimos del interesado", siendo estas garantías, "como mínimo el derecho a obtener intervención humana por parte del responsable, a expresar su punto de vista y a impugnar la decisión"36.

La doctrina ha interpretado, conforme a este artículo, que existe una obligación del responsable de datos de informar al afectado de las razones que han llevado a tomar esa decisión. Es decir, la empresa, cuando tome decisiones automatizadas, deberá indicarlo e informar al trabajador individual de qué parámetros (y qué ponderación ha asignado a cada uno de ellos) ha utilizado para alcanzar dicha resolución. Esta interpretación es apoyada por el artículo 5 RGPD que exige que el procesamiento de datos sea legal, justo y trasparente y, también, por los artículos 13.2 f) y 14.2 g) RGPD que exigen que cuando el sujeto es objeto de decisiones automatizadas, incluyendo la elaboración de perfiles, el

\footnotetext{
${ }^{34}$ GT29, en Guidelines on transparency under Regulation 2016/679, p. 23.

${ }^{35}$ El considerando 43 del RGPD establece que no es posible aceptar la licitud para el tratamiento y procesamiento de datos en base al consentimiento en una relación donde exista un fuerte desequilibrio de poder entre las partes. Concretamente, el Reglamento hace referencia a la relación entre Administraciones Públicas y ciudadanos, no obstante, esta consideración parece perfectamente aplicable a la relación laboral. De hecho, el Grupo de Trabajo del art 29, en su Guía sobre el consentimiento bajo el RGPD (p. 8) entiende que el consentimiento de un trabajador difícilmente puede ser otorgado cumpliendo dichos requisitos (específicamente de forma "libre"). Por esta razón, el Grupo de Trabajo dictamina que, dada la naturaleza de la relación entre empresario y trabajador, como regla general, no se deberá entender válido el otorgamiento de consentimiento por parte del trabajador, teniendo que ser solamente aceptado como válido de forma excepcional.

${ }^{36}$ Ver más en TODOLí SigNES, Adrián, "La gobernanza colectiva de la protección de datos en las relaciones laborales: big data, creación de perfiles, decisiones empresariales automatizadas y los derechos colectivos", $R D S, \mathrm{n}^{\circ} 84,2018$, p. 69-88.
} 
responsable de datos deberá entregar al sujeto información significativa sobre la lógica aplicada, así como la importancia y las consecuencias previstas de dicho tratamiento para el interesado.

De esta manera, se comprueba que la configuración como derecho colectivo dentro del derecho nacional es de mayor alcance: se aplica a un superior número de supuestos que la regulación europea de carácter individual. Sin embargo, esta última, cuando surte efecto, tiene un contenido mayor que no consiste en el mero derecho de información, sino que permite al trabajador individual exigir la intervención humana en la decisión tomada por el algoritmo, así como, el derecho del trabajador a expresar su punto de vista.

\subsection{Contenido de la información a entregar}

El nuevo artículo 64.4 d) ET establece que el comité de empresa tendrá derecho a ser informado de "los parámetros, reglas e instrucciones en los que se basan los algoritmos o sistemas de inteligencia artificial'. Una vez más, esta norma se aparta de la redacción de la normativa europea que establece el derecho individual del trabajador afectado a conocer la información significativa "sobre la lógica aplicada, así como la importancia y las consecuencias previstas de dicho tratamiento para el interesado".

Respecto a la normativa europea, la doctrina, de manera unánime, interpreta que el interesado tiene derecho a recibir información sobre el uso de los algoritmos en tres aspectos: i) informar de que el sujeto está envuelto en un proceso automatizado de toma de decisiones, es decir, informar al trabajador de que el proceso de decisión será total o parcialmente automatizado; ii) proveer información significativa sobre la lógica del algoritmo, esto es, entre otras cuestiones, indicar los parámetros evaluados por el algoritmo que toma la decisión y la ponderación de dichos parámetros; iii) informar de las consecuencias del proceso, es decir, qué consecuencias tendrá para el trabajador la decisión, en un sentido u otro, tomada de forma automática ${ }^{37}$.

\footnotetext{
${ }^{37}$ WACHTER, Sandra, Brent, MitTelstadt, Luciano, FloRIDI, "Why a Right to Explanation of Automated Decision-Making Does Not Exist in the General Data Protection Regulation" International Data Privacy Law, n 7, 2017, p. 79-90; MendozA, Isak, and BYgRAVE, Lee, "The Right Not to Be Subject to Automated Decisions Based on Profiling", en SYNODINOU, Tatiani, et al. (eds.,) EU internet law: regulation and enforcement, 2017, https://papers.ssrn.com/abstract=2964855 [https://perma.cc/XV3T-G98W]; EDWARDS, Lilian, and VEALE, Michael, "Slave to the Algorithm?..." op cit., p. 18-82; WAN, Kim, RouTLEDGE, Bernad, "Algorithmic Transparency, a Right to Explanation, and Placing Trust", Squarespace, 2017, https://static1.squarespace.com/static/592ee286d482e908d35b8494/t/59552415579fb3 0c014cd06c/1498752022120/Algorithmic+transparency\%2C+a+right+to+explanation+ and+trust+\%28TWK\%26BR\%29.pdf [https://perma.cc/K53W-GVN2, visitado 07/05/2021; MALGIERI, Gianclaudio, and COMANDÉ, Giovanni, "Why a Right to Legibility of Automated DecisionMaking Exists in the General Data Protection Regulation", International data privacy Law, vol. 7, n 4, 2017, p. 243-
} 
Sin embargo, la doctrina no es unánime a la hora de interpretar el grado de explicación necesaria. Por una parte, una minoría de autores establecen que la obligación se limita a exigir una explicación ex ante (anterior a la toma de la decisión) y general de los datos suministrados al algoritmo para que este tome la decisión ${ }^{38}$. Por su parte, la doctrina mayoritaria establece que el artículo 22.3 RGPD, en conjunción con los artículos 13.2 f) y $14.2 \mathrm{~g}$ ) y el considerando 71 del RGPD, exigen una explicación ex post (después de tomar la decisión) y específica de cómo y porqué se ha tomado dicha decisión sobre ese concreto trabajador ${ }^{39}$.

Respecto al nuevo artículo 64.4 d) ET no parece que vaya a repetirse dicho debate. En efecto, dado que los derechos de información de los representantes de los trabajadores son generales sobre el uso del algoritmo y no respecto a una concreta decisión -o usoindividual del algoritmo, parece que los derechos de información se limitarán a una explicación ex ante (anterior a al uso del algoritmo para supuestos concretos) y general de los parámetros reglas e instrucciones con los que el algoritmo está diseñado para que este tome decisiones.

Una cuestión sobre la que la normativa nacional guarda silencio es sobre si la empresa deberá informar a los representantes de los trabajadores del uso que se vaya a dar a ese algoritmo y de las consecuencias que pueda tener. Esto es, se plantea la duda de si la empresa estará obligada a informar respecto a si el algoritmo se usará para procesos de selección, evaluación de trabajadores, ascensos, despidos, etc., o cuál será el nivel de intervención del algoritmo en estas materias.

265; GoOdman, Bryce, and Flaxman, Seth, "EU Regulations on Algorithmic Decision-Making and a "Right to Explanation," ARXIV, no 6, 2016, http://arxiv.org/abs/1606.08813 [https://perma.cc/5ZTRWG8R]; SElbst, Andrew, Powles, Julia, "Meaningful information and the right to explanation", International Data Privacy Law, n 233, 2017, WACHTER, Sandra, et al, "Counterfactual explanations without opening the black box: automated decisions and the GDPR", Arxiv, 2017, p. 1-51, en https://arxiv.org/ftp/arxiv/papers/1711/1711.00399.pdf; GONZALEZ, GIL, Elena “Aproximación al estudio de las decisiones automatizadas en el seno del Reglamento General Europeo de Protección de Datos a la luz de las tecnologías big data y de aprendizaje computacional", Revista española de la Trasparencia, $\mathrm{n}^{\circ}$ 5, 2017, p. 165-179.

${ }^{38}$ WACHTER, Sandra, MitTELSTADT, Brent, FLORIDI, Luciano, "Why a Right to Explanation of Automated Decision-Making Does Not Exist in the General Data Proteccion Regulation" International Data Privacy Law, no 7, 2017, p. 79-90; WACHTER, Sandra, et al., "Counterfactual...”, op cit, p. 1-51, GIL GONZALEZ, Elena, “Aproximación...", op cit., p. 165-179.

${ }^{39}$ MendoZA, Isak, and Bygrave, Lee, “The Right Not to Be Subject...”, op cit., EdwARds, Lilian, and VEALE Michael, "Slave to the Algorithm?" op cit., p. 18-82; WAN, Kim, and RoUTLEDGE, Bernad, "Algorithmic Transparency, op cit., visitado 07/05/2021; MALGIERI, Gianclaudio, and COMANDÉ, Giovanni, "Why a Right to Legibility...", op cit.; GOODMAN, Bryce, FLAXMAN, Seth, "EU Regulations on Algorithmic...”, op cit., SELBST, Andrew, and POWLES, Julia, “Meaningful information...”, op cit., GINÈS I FABRELLAS, Anna, “¿Tienen $1 @$ s riders derecho a conocer el algoritmo?, en Do Better, 2021. Accessible en https://dobetter.esade.edu/es/riders-derecho-algoritmo. 
Sobre esta cuestión cabe decir que difícilmente se puede separar una información de otra. La información entregada a los representantes de los trabajadores sobre los parámetros, reglas e instrucciones del algoritmo, con objeto de que pueda ser de alguna utilidad, debe entregarse en su contexto. Lo cual incluye, al menos, para qué se usará ese algoritmo.

Ciertamente, cualquier análisis sobre los riesgos que pueda tener el algoritmo -riesgos de discriminación ${ }^{40}$, riesgos para la salud de los trabajadores ${ }^{41}$, etc., partirá de conocer para qué se va a usar -qué decisiones va a tomar el algoritmo- y con qué intensidad se va a usar- ¿tomará decisiones sin intervención significativa de un ser humano?, ¿se usará solamente como apoyo a la toma de decisiones?, ¿irá acompañado de auditorías "humanas"?

Lo que no parece que puedan exigir los representantes de los trabajadores con la redacción actual es información sobre todas y cada una de las decisiones tomadas por el algoritmo a posteriori (una vez tomadas). Un vacío que dificultará que los representantes de los trabajadores puedan analizar y evaluar el buen funcionamiento del propio algoritmo con objeto de poder garantizar que este no discrimina, no vulnera derechos fundamentales o, sencillamente, que no está tomando decisiones arbitrarias. En cualquier caso, nada obsta para que esto se pacte en la negociación colectiva o se incluya posteriormente en la ley. Adicionalmente, los trabajadores individuales afectados sí podrán reclamarlo vía artículo 22 RGPD o los representantes en su nombre como he defendido en otros lugares ${ }^{42}$.

Tampoco parece que la redacción actual del nuevo apartado d) del artículo 64 ET obligue a la empresa a identificar qué información ha suministrado al algoritmo para que pueda tomar sus decisiones. Los algoritmos, con objeto de poder establecer correlaciones en los datos, que le permitan posteriormente tomar decisiones, necesitan "alimentarse" con gran cantidad de información previas, momento en el que el algoritmo "aprenderá". Tal y como señala la doctrina ${ }^{43}$, la calidad de estos datos será clave para asegurar que el algoritmo no discrimina y es que, si se "alimenta" al algoritmo con datos sesgados, el algoritmo

\footnotetext{
${ }^{40}$ HARDT, Moritz, "How big data is unfair", Medium 2014, https://medium.com/@mrtz/how-big-data-isunfair-9aa544d739de; RIVAS VALLEJO, María Pilar, La aplicación de la inteligencia artificial al trabajo y su impacto discriminatorio, Aranzadi, 2020.

${ }^{41}$ Todolí Signes, Adrián, "En cumplimiento de la primera Ley de la robótica: Análisis de los riesgos laborales asociados a un algoritmo artificial / inteligencia dirigiendo el trabajo", Labor Law \& Issues, vol. $5, \mathrm{n}^{\circ}$ 2, 2019, disponible en https://papers.ssrn.com/sol3/papers.cfm?abstract_id=3506584.

42 TODOLí SigNES, Adrián, "La gobernanza colectiva de la protección de datos en las relaciones laborales: big data, creación de perfiles, decisiones empresariales automatizadas y los derechos colectivos", $R D S, \mathrm{n}^{\circ}$ 84, 2018, p. 69-88.

${ }^{43}$ HARDT, Moritz, "How big data is unfair", Medium 2014, https://medium.com/@ mrtz/how-big-data-isunfair-9aa544d739de.
} 
entenderá que discriminar es la mejor forma de tomar decisiones. Así pues, de nuevo, sin un conocimiento claro de los datos usados por la empresa para "entrenar" al algoritmo será difícil analizar el buen funcionamiento del mismo.

\subsection{Derechos de información ya existentes que afectan a los algoritmos}

Es importante señalar que los derechos de información sobre el algoritmo o sistema de inteligencia artificial no terminan con el nuevo apartado d) del artículo 64.4 ET. El resto de derechos de información de los representantes de los trabajadores siguen vigentes y serán aplicables cuando se use un algoritmo para tomar decisiones.

Así, el artículo 64.5 ET establece que el comité de empresa "tendrá derecho a ser informado y consultado sobre todas las decisiones de la empresa que pudieran provocar cambios relevantes en cuanto a la organización del trabajo". Así pues, el uso de un algoritmo que afecte a la organización del trabajo dará derecho al comité, no solo a ser informado sobre el mismo y su funcionamiento, sino también obligará a la empresa a consultar a los representantes antes de su puesta en funcionamiento.

De la misma manera, conforme al artículo 64.5 f) ET, si el algoritmo tiene incidencia sobre los sistemas de organización y control del trabajo -incluyendo poder disciplinario, los sistemas de rendimiento, de tiempo de trabajo o de primas o incentivos, el comité de empresa tendrá derecho a ser informado y a emitir un informe previa su puesta en funcionamiento.

En ambos casos, para que se cumplan los derechos de consulta y emisión de informe es necesario que la información entregada sea apropiada y adecuada conforme el artículo 64.6 ET. Así pues, al menos en estos casos, sí parece que el comité de empresa pueda solicitar información, una vez puesto en marcha el algoritmo, sobre las decisiones que está tomando y su funcionamiento con objeto de valorar que no está discriminando y, en general, que no está vulnerando ningún derecho de los trabajadores. Esta información respecto a las consecuencias de las decisiones tomadas por el algoritmo -incluido los perfiles- debería entregarse en un formato adecuado para su tratamiento por parte de los representantes. De la misma forma, en estos casos, parece que sí sería exigible que los representantes de los trabajadores conocieran qué datos han sido suministrados (o lo van a ser) al algoritmo para su desarrollo.

\subsection{Trascendencia del nuevo derecho de información}

De forma similar a como ocurre con la presunción de laboralidad de la DA23, que ya existía de forma general en el artículo 8.1 ET, el nuevo derecho de información de los 
representantes de los trabajadores, probablemente, pudiera haberse extraído del resto de derechos de información ya existentes en el Estatuto de los trabajadores. Sin embargo, se debe aplaudir que el legislador no haya esperado a que el Tribunal Supremo se hubiera pronunciado sobre esta cuestión y que, por el contrario, demuestre verdadera iniciativa propia regulando una cuestión actual, adelantándose, así, a la conflictividad.

En la práctica, uno de los mayores problemas que puede surgir en materia de algoritmos es el desconocimiento total que pueden tener los representantes de los trabajadores y los propios trabajadores de su existencia. No es solo que la novedad de su uso haga difícil que estos estén informados, sino que, además, la intangibilidad del algoritmo puede hacer imposible a la parte social el conocimiento de su mera existencia. De esta forma, parece muy relevante que la norma, de forma clara, exponga la obligación de informar, no solo a los propios trabajadores (artículo 22 RGPD), sino también a los representantes de los trabajadores (nuevo artículo 64.4 d)) ET de su existencia.

La doctrina ha señalado en multitud de ocasiones los perjuicios que puede tener para el trabajador el uso de estos sistemas como sustituto de los supervisores humanos discriminación, deshumanización, vulneración de derechos fundamentales, problemas de salud- ${ }^{44}$. Por ello, cualquier medida que traiga trasparencia sobre su uso debe ser elogiada.

No obstante, este -el conocimiento de su existencia- no deja de ser solamente el primer paso. Dado que los algoritmos pueden ser determinantes para los sistemas de trabajo, las condiciones de trabajo, la contratación, los ascensos y los despidos, parece obvio que el siguiente paso será negociar colectivamente su funcionamiento ${ }^{45}$. En el fondo, esto no

\footnotetext{
${ }^{44}$ MOORE, Phoebe, Work and the GDPR: The future for algorithms and people analytics, 2017, available at https://staffblogs.le.ac.uk/management/2018/09/20/work-and-the-gdpr-the-future-for-algorithms-andpeople-analytics/; MOORE, Phoebe, The quantified self in precarity. Work, technology and what counts, New York, Routledge, 2018; MOORE, Phoebe, Humans and machines at work: monitoring, surveillance and automation in contemporary capitalism, London. Palgrave Macmillan, 2018; MoORE, Phoebe, Data subjects, digital surveillance, AI and the future of work, European Parliament Science and Technology Office, Brussels, 2020; UTS, Heat Stress and On-Demand work: The experience of food delivery and courier cyclists. Climate Justice Centre, 2019, disponible en https://opus.lib.uts.edu.au/bitstream/10453/134736/1/On\%20Demand\%20cyclists\%20UTS\%20final.pdf; TODOLÍ SIGNES, Adrián, "En cumplimiento de la primera Ley de la robótica: Análisis de los riesgos laborales asociados a un algoritmo artificial / inteligencia dirigiendo el trabajo", Labor Law \& Issues, vol. 5, $\mathrm{n}^{\mathrm{o}}$ 2, 2019, disponible en https://papers.ssrn.com/sol3/papers.cfm?abstract_id=3506584; RIVAS VALLEJO, María.Pilar., La aplicación de la inteligencia artificial al trabajo y su impacto discriminatorio, Aranzadi, 2020.

${ }^{45}$ De Stefano, Valerio, "'Negotiating the Algorithm": Automation, Artificial intelligence and labour protection", Comparative Labor Law \& Policy Journal, 2018; TODOLí SigNES, Adrián, "La gobernanza colectiva de la protección de datos en las relaciones laborales: big data, creación de perfiles, decisiones empresariales automatizadas y los derechos colectivos", $R D S, \mathrm{n}^{\circ}$ 84, 2018, p. 69-88. Así se pronuncia
} 
será otra cosa que cumplir con la función clásica de la negociación colectiva: negociar colectivamente las condiciones de trabajo.

\section{Entrada en vigor a los tres meses de su publicación}

El Real Decreto-ley 9/2021, de 11 de mayo establece que "entrará en vigor a los tres meses de su publicación en el Boletín Oficial del Estado". Esta cuestión, además de poner en duda de que su aprobación sea de "extraordinaria y urgente" necesidad conforme a las exigencias constitucionales para este tipo de normas, plantea qué ocurre con la situación de los trabajadores hasta su entrada en vigor.

En este sentido, podría interpretarse como una "amnistía" en favor de las empresas hasta la entrada en vigor tres meses después de su publicación. Esto es, cabría entender que, dado que la presunción de laboralidad de los trabajadores en plataformas no entra en vigor hasta el 12 de agosto, ello implica que las empresas no tendrán obligación de contratar como laborales a los repartidores hasta esa fecha.

Sin embargo, a mi juicio, esa interpretación no encajaría con la opción legislativa tomada. En efecto, como se ha dicho, la nueva presunción incorporada no modifica sustantivamente el concepto de asalariado. De esta forma, quien era trabajador asalariado un día antes de la entrada en vigor de esta nueva normativa, lo seguirá siendo un día después, al igual que quien no era trabajador laboral, no pasará a serlo materialmente por la entrada en vigor de esta normativa. Ello significa que el retraso en la entrada en vigor marcado por la normativa no afecta a la realidad material existente.

De hecho, los repartidores de plataformas tendrán derecho a que se les reconozca la antigüedad desde el inicio de sus actividades en la plataforma -salvo que la empresa de plataformas pueda demostrar que un determinado repartidor incurría en una de las exclusiones del artículo 1.3 ET-. Se llega a esta conclusión dado que se debe entender, conforme a la STS de 25 de septiembre de 2020 y la nueva presunción, que desde el inicio debieron estar clasificados como asalariados.

También, la Tesorería General de la Seguridad Social, a través de la Inspección de trabajo, podrá solicitar las cotizaciones no ingresadas en el régimen general por todos estos repartidores que hasta el momento estaban dados de alta como autónomos pero que solamente ahora son reclasificados (salvo las prescritas). Esta responsabilidad

también el ROJO TORRECILLA, Eduardo, "Y llegó...", op cit, en los siguientes términos "abriéndose así un amplio espacio para, más allá de la mera información, empezar a debatir y negociar sobre "la gestión del algoritmo". 
correspondería desde el inicio de la prestación de servicios de repartidor como autónomo hasta su correcta clasificación como laboral ${ }^{46}$.

\section{Valoración final}

La normativa que aquí se analiza tiene aspectos muy positivos y otros no tanto. En los aspectos menos positivos se deben resaltar tres. De un lado, el limitadísimo alcance de la nueva presunción, que se circunscribe solamente al sector del reparto en plataformas digitales, dejando fuera el resto de los sectores de plataformas digitales. Esto implica que la norma no consigue poner remedio al famoso fenómeno de "huida del Derecho del trabajo" mediante un uso fraudulento de la figura del autónomo. De hecho, como señalan los estudios de la Comisión Europea ${ }^{47}$, el transporte en plataformas equivale a menos del $5 \%$ de todo el trabajo en plataformas, por lo que esta regulación deja tal y como estaba con los niveles de inseguridad jurídica, de conflictividad y de precariedad- la inmensa mayoría de trabajadores y empresas de plataformas digitales. Por el contrario, otras regulaciones como la ley AB5 de California, mucho más ambiciosas, han extendido su regulación a todos los sectores -con algunas excepciones expresas- sean o no de plataformas $^{48}$.

El segundo aspecto que puede criticarse es la técnica legislativa usada. De un lado, debido a que ya existe una presunción de laboralidad en el artículo 8.1 ET, la nueva presunción específica, si se entiende como una presunción iuris tantum, no aporte mucho al contenido jurídico del ET. Si entendemos que la nueva presunción es una presunción iuris et de iure sí se estaría aportando contenido real. Sin embargo, dada la redacción de la DA23 ET no queda claro que esta fuera la intención por lo que, de nuevo, surgirán las dudas interpretativas generando inseguridad jurídica y obligando a acudir a juicio a quién desee demostrar que se está ante un trabajador laboral. Esto es, una situación muy parecida a la que nos encontrábamos antes de la inclusión de esta DA 23 ET. Lo que sí parece que

\footnotetext{
${ }^{46}$ Una vez más, salvo que la empresa pueda acreditar que, sobre un repartidor concreto, concurre alguna de las excepciones del artículo 1.3 ET.

${ }^{47}$ Urzí Brancati, María Cesira, Pesole, Annarosa, Fernández-Macías, Enrique, "New evidence on platform workers in Europe. Results from the second COLLEEM survey", EUR 29958 EN, Publications Office of the European Union, Luxembourg, 2020.

48 TodOlí SIGNES, Adrián "El ámbito subjetivo de aplicación del Estatuto de los Trabajadores ante las nuevas formas de trabajo: la nueva ley de California (AB5)", TyD, nº6, 2020. En efecto, la cuestión de los "falsos autónomos" plantea retos más allá del sector del trasporte y el reparto que necesitan soluciones globales, al respecto ver algunas propuestas en RODRÍGUEZ FERNÁNDEZ, María Luz, Protección social para los trabajadores de la economía de plataforma propuestas para aliviar su vulnerabilidad", Revista General de Derecho del trabajo y de la Seguridad Social, no 57, 2020; Mercader Uguina, Jesús, Nuevos escenarios para el Estatuto de los Trabajadores del siglo XXI. Digitalización y cambio tecnológico", $T y D$, $n^{\circ} 63,2020$.
} 
aporte esta nueva normativa es la elevación a rango legal la rebaja en la exigencia en el cumplimiento del requisito de dependencia jurídica para entender que se está ante un trabajador laboral. De esta forma, parece que siempre que se dirija o coordine el trabajo del repartidor a través de un algoritmo habrá que entender que se está dentro de los conceptos de dependencia y ajenidad, admitiendo solamente argumentos que justifiquen la exclusión de la laboralidad con un fundamento distinto a la falta de dependencia y ajenidad.

Adicionalmente, se puede criticar que, habiendo abierto el "melón" legislativo, no se haya aprovechado para proponer alguna solución a la cuestión de los horarios y la jornada en el trabajo en plataformas -aunque fuera para los repartidores- ${ }^{49}$. En España, el contrato "a llamada" o de "cero horas" no es lícito ${ }^{50}$. Esto implica que las plataformas deberán garantizar a todos los repartidores un mínimo de horas, no pudiendo "convocar" a los trabajadores según demanda. Esta parte de la regulación, que puede considerarse positiva para algunos de los trabajadores, tiene la contraparte negativa: los trabajadores perderán flexibilidad -si actualmente tuvieran alguna- a la hora de tener la facultad de elegir cuántas horas trabajan y en qué horario. Una flexibilidad que podría ser positiva para algunos de ellos.

Con la aplicación de la normativa laboral, las empresas deberán determinar por adelantado, conforme lo regulado en el Estatuto de los trabajadores, la jornada mínima del trabajador y los horarios de estos con antelación, a los que los trabajadores deberán comprometerse quedando obligados a cumplir con el horario determinado por la empresa $^{51}$. Esto podría llegar a implicar que aquellos trabajadores que necesiten flexibilidad horaria y de jornada (por otros trabajos, estudios, conciliación, etc.) no puedan seguir en la plataforma a partir de la laboralización. Como se ha defendido en otros lugares ${ }^{52}$, el trabajo en plataformas aporta algunas ventajas para los trabajadores: la regulación debería eliminar la precariedad, pero sin desbaratar aquellas cuestiones

\footnotetext{
${ }^{49}$ No obstante, cabe señalar que parte de la doctrina ha defendido que no debería haber modificación normativa alguna en materia de tiempo de trabajo en el trabajo en plataformas. Por el contrario, las empresas deben cambiar su modelo de negocio para adaptarlo a la legislación vigente en materia de horarios, ver GiNÈS I FABRELLAS, Anna., "El tiempo de trabajo en plataformas: ausencia de jornada mínima, gamificación e inseguridad algorítmica", Labos, vol. 2, nº 1, 2021, p. 19-42.

${ }^{50}$ GINÈS I FABRELLAS Anna., "The zero-hour contract in platform work. Should we ban it or embrace it?, IDP Revista de internet, Derecho y Política, 28, 2019, p. 1-15.

${ }^{51} \mathrm{El}$ tema de los horarios en el trabajo en plataformas ha centrado el interés de la doctrina dado su complejo encaje, ver al respecto RODRÍGUEZ RODRÍGUEZ, Emma, "La transcendencia de la disponibilidad horaria del trabajador en el contexto de las plataformas digitales", Temas Laborales, $\mathrm{n}^{\circ}$ 146, 2019, p. 121-158; GIMENO DíAZ DE ATAURI, Pablo, "Problemas prácticos para la ordenación del tiempo de trabajo en las relaciones laborales de plataformas digitales de reparto", Documentación Laboral, n 121, 2020, p. 105-118.

${ }^{52}$ Todolí Signes, Adrián, El trabajo en la era de la economía colaborativa, Tirant lo Blanch, 2017.
} 
provechosas que pueda conllevar. En este sentido, se podría haber regulado expresamente el derecho de los repartidores a elegir su horario y jornada de trabajo sin poder sufrir represalias por parte de la empresa cuando rechazan encargos realizados por la empresa fuera de la jornada elegida por el trabajador ${ }^{53}$.

Tampoco se ha aprovechado la normativa específica para poner solución a la cuestión de la "concurrencia desleal" en el contrato de trabajo. El trabajo en plataformas, como forma de organización del trabajo, facilita que los repartidores presten servicio en más de una empresa de reparto. Dentro del régimen jurídico laboral, esto se consideraría concurrencia desleal (artículo $21 \mathrm{ET}$ ) por parte del trabajador, lo que, salvo que las empresas de plataforma permitan expresamente la compatibilidad, implicará que los trabajadores de plataformas tengan que elegir prestar servicios solamente en una de ellas, con la correspondiente reducción de las posibilidades de obtener más trabajo. A mi juicio, hubiera tenido más sentido aprovechar la normativa para establecer una excepción al régimen actual en materia de "concurrencia desleal" para los trabajadores de plataformas, permitiendo la compatibilidad para trabajar en más de una plataforma de reparto (salvo pacto expreso en contrario) $)^{54}$.

Los aspectos positivos de la nueva regulación superan los vistos hasta ahora. De hecho, algunos de los aspectos negativos parecen inseparables de los positivos. Así ocurre con el hecho de que la regulación sea fruto del diálogo social. Esta podría considerarse una de las grandes ventajas de la regulación por varios motivos.

En primer lugar, coloca a España como un referente a nivel mundial en materia de solución negociada al "problema" de las plataformas digitales. Otros países han regulado la cuestión, pero ninguno lo ha hecho mediante el consenso y el diálogo social.

En segundo lugar, que la regulación provenga del diálogo social le da un plus de efectividad. De un lado, la aquiescencia de la patronal a esta regulación desmonta los argumentos de determinadas empresas que sostenían que, con la laboralización, el sector desaparecería al hacer inviable el modelo de negocio. Estas empresas han quedado deslegitimadas con la actuación de la patronal. Así, con la aceptación de esta regulación, se demuestra indirectamente que estas declaraciones no tienen sustento suficiente ni son mayoritarias dentro de los propios empresarios.

\footnotetext{
${ }^{53}$ Propuesta que ya realizaba en TODOLÍ SIGNES, Adrián, El trabajo en la era de la economía colaborativa, Tirant lo Blanch, 2017.

54 Propuesta que ya se realizaba en Todolí SignES, Adrián, "El Impacto de la "Uber economy" en las relaciones laborales los efectos de las plataformas virtuales en el contrato de trabajo", iuslabor, 2015, disponible para descargar en https://adriantodoli.com/2015/12/22/el-impacto-de-la-uber-economy-en-lasrelaciones-laborales-los-efectos-de-las-plataformas-virtuales-en-el-contrato-de-trabajo-2/.
} 
En tercer lugar, la norma, y su origen en el diálogo social, confiere un "espaldarazo" a la interpretación del concepto de trabajador que viene haciendo en los últimos años el Tribunal Supremo. Como se ha señalado en este comentario, el Tribunal Supremo viene concediendo cada vez menos importancia al poder de dirección y control como requisito necesario de la laboralidad. Actualmente, esto ya no se encuentra solo en las resoluciones del Tribunal Supremo, sino que, ahora, esta reinterpretación del concepto de trabajador tiene el apoyo del diálogo social y de la propia ley. Sin duda, esto puede dar alas al Tribunal Supremo para continuar en esta línea interpretativa con objeto de conseguir la deseada adaptación del concepto de trabajador a las formas organizativas del S. XXI que, en mi opinión, debe pasar por la progresiva desaparición del criterio de dependencia jurídica como requisito para la aplicación del Derecho del trabajo.

En fin, esta norma se enmarca dentro de la tendencia de los últimos años de regular, no con objeto de innovar en materia de Derechos laborales, sino como forma de mejorar la efectividad de derechos que ya se tenían. Así ha pasado con la obligación del registro horario, del registro salarial, con el derecho a la desconexión digital y, en cierta medida también, con la ley del teletrabajo. Concretamente, con la digitalización se opta, no está claro si acertadamente, por modificar la norma para incluir nuevas disposiciones que concretan, especifican o aclaran la aplicabilidad de los derechos en el mundo digital.

Así, esta norma pretende cerrar el debate sobre la aplicabilidad de los derechos laborales en el trabajo en plataformas. En este sentido, la norma implica un posicionamiento, de los agentes sociales y del Gobierno, en contra del "determinismo tecnológico". Esto es, no existe nada inherente a las nuevas tecnologías que las haga incompatible con la regulación laboral. De esta forma, aunque se haya quedado solo en el sector del reparto, la realidad es que la redacción incita a pensar que el sector no es lo relevante: lo importante es haber determinado que las nuevas posibilidades tecnológicas -algoritmos y plataformas digitales- no son más que los medios de producción por los que se ejerce una actividad económica y, por ello, los que prestan servicios dentro de su ámbito deberán considerarse trabajadores laborales.

\section{Bibliografía}

AJUnwA, Ifeoma, et al., "Limitless worker surveillance", California Law Review, vol. $105, n^{\circ} 3,2017$.

AkHtar, Jam, and Moore, Phoebe, "The Psycho-Social Impacts of Technological Change in Contemporary Workplaces and Trade Union Responses", International Journal of Labor Research, vol. 8, no 1-2, 2016, p. 102-131. 
Alarcón CARACUEl, Manuel Ramon, "La ajenidad en el mercado: Un criterio definitorio del contrato de trabajo", Civitas, n $28,1986$.

Albiol MonTEsinos, Ignacio, "Entorno a la polémica ajenidad-dependencia", $C C D T, \mathrm{n}^{\circ}$ $1,1971$.

ALOISI, Antonio, "A fascinating chapter in the "gig" saga. How to deliver decent work to platform workers in Italy?", Mutual learning Programme, DG Employment, Social Affairs and Inclusion, European Union, 2020; https://ec.europa.eu/social/main.jsp?catId=89\&furtherNews=yes\&newsId=9746\&langId $=$ en\#navItem -1

Alonso Olea, Manuel, Introducción al Derecho del Trabajo, ed. Revista de Derecho Privado, Madrid, 1968 Montoya Melgar, Alfredo, "El poder de dirección del empresario", IEP, Madrid 1965.

Álvarez Alonso, Diego, y Martínez Moreno, Carolina, "Trabajo y plataformas digitales primera sentencia del Tribunal Supremo a propósito de un repartidor de Glovo", $T y D, \mathrm{n}^{\circ} 72,2020$.

BAYLOS GRAU, Antonio, "Por fin la norma sobre los repartidores de plataformas (la ley "riders”)", Según Antonio Baylos, 2021.

BAyón Chacón, Gaspar, PÉRez Botida, Eugenio, Manual de Derecho del Trabajo, Marcial Pons, Madrid, 1976.

CABEZA PEREIRO, Jaime, El ámbito del trabajo subordinado y del trabajo autónomo en el Derecho de la Unión Europea, Bomarzo, 2020.

CONGER, Kate, "Uber and Lyft Drivers in California Will Remain Contractors", New York Times, https://www.nytimes.com/2020/11/04/technology/california-uber-lyft-prop22.html

Countouris, Nicola, De Stefano, Valerio, Lianos, Ionnis "The EU, competition and worker's Rights", Center for Law, Economics and Society, n 2, 2021.

CRUZ VILlalón, Jesús., "Una presunción plena de laboralidad de los riders", Blog de Cruz Villalón, 2021. 
De La ViLla GiL, Luis Enrique, El trabajo a domicilio, Aranzadi, Madrid, 1966.

DE Stefano, Valerio "The EU Proposed Regulation on AI: a threat to labour protection?", Regulation for Globalization, 2021.

DEMBE, Aron, et al., "The impact of overtime and long work hours on occupational injuries and illnesses: new evidence from the United States", Occupational and Environmental Medicine, vol. 62, no 9, 2005, p. 588-597.

DERKS, Daantje, and BAKKER, Arnold B., "Smartphone use, work-home interference and burnout: a diary study on the role of recovery", Applied Psychology, vol' 63 (3), 2014.

DOMEINSKI, Juliane, et al., "Human redundancy in automation monitoring: Effects of social loafing and social compensation", en Proceeding of human factors and ergonomics Society 51st Annual meeting, 2007, p. 587-591.

EU-OSHA, Foresight on new and emerging occupational safety and health risks associated with digitalisation by 2025, 2018 disponible en https://osha.europa.eu/en/tools-and-publications/publications/foresight-new-andemerging-occupational-safety-and-health-risks/view

EU-OSHA, Monitoring technology: the 21st century's pursuit of well-being?, 2017 disponible en https://osha.europa.eu/en/tools-and-publications/publications/monitoringtechnology-workplace/view (Accessed 29/08/2019).

FERNÁNDEZ AvILÉS, José Antonio., "NTIC y riesgos psicosociales en el trabajo: estado de situación y propuestas de mejora", DSL, nº 2, 2017.

Fundación Felipe González, Huella Digital: La plataformización del trabajo en Europa, 2019.

GIL GonZALEZ, Elena, “Aproximación al estudio de las decisiones automatizadas en el seno del Reglamento General Europeo de Protección de Datos a la luz de las tecnologías big data y de aprendizaje computacional", Revista española de la Trasparencia, $\mathrm{n}^{\mathrm{o}} 5$, 2017, p. 165-179.

Gimeno Díaz DE ATAURI, Pablo, "Problemas prácticos para la ordenación del tiempo de trabajo en las relaciones laborales de plataformas digitales de reparto", Documentación Laboral, $\mathrm{n}^{\circ} 121,2020$, p. 105-118. 
GINÈS I FABRELlAS, Anna, “¿Tienen $1 @$ s riders derecho a conocer el algoritmo?, en Do Better, 2021. Accesible en https://dobetter.esade.edu/es/riders-derecho-algoritmo

GINÈS I FABRELLAS, Anna, "El tiempo de trabajo en plataformas: ausencia de jornada mínima, gamificación e inseguridad algorítmica”, Labos, vol. 2, nº 1, 2021, p. 19-42.

GINÈS I FABRELLAS, Anna, "The zero-hour contract in platform work. Should we ban it or embrace it?, IDP Revista de internet, Derecho y Política, n 28, 2019, p. 1-15.

Goerlich Peset, Jose Maria y López Terrada, Eva, "Cambios productivos, reestructuraciones y negociación colectiva”, Gaceta Sindical, no 32, 2019.

Goerlich Peset, Jose Maria y García Rubio, María Amparo, "Indicios de autonomía y de laboralidad en los servicios de los trabajadores en plataforma", en PÉREZ DE LOS CoBos ORIHUEL, Francisco (Coord.), El trabajo en plataformas digitales, Wolters Kluwer, 2018.

Goerlich PeSet, Jose Maria y GARCÍA Rubio, María Amparo, "Indicios de autonomía y de laboralidad en los servicios de los trabajadores en plataforma”, en PÉREZ DE LOS CoBos ORIHUEL, Francisco (Coord.), El trabajo en plataformas digitales, Wolters Kluwer, 2018.

Goodman, Bryce, and Flaxman, Seth, "EU Regulations on Algorithmic DecisionMaking and a "Right to Explanation," ARXIV, n' 6, 2016, http://arxiv.org/abs/1606.08813 [https://perma.cc/5ZTR-WG8R].

Gorelli Hernández, Juan, "Paso a paso hacia el Tribunal Supremo el problema de la laboralidad de la prestación de servicios en plataformas digitales (casos Glovo y Deliveroo)", $T y D$, n $^{\circ} 60,2019$, p. 75-88.

GT29 en Guidelines on Automated individual decision-making and Profiling for the purposes of Regulation 2016/679, Adoptadas el 3 de octubre de 2017.

GT29, en Guidelines on transparency under Regulation 2016/679.

HARDT, Moritz, "How big data is unfair", Medium 2014, https://medium.com/@mrtz/how-big-data-is-unfair-9aa544d739de 
HORTON, John, et al., "Workplace Safety Futures: The impact of emerging technologies and platforms on work health and safety and workers' compensation over the next 20 years", CSIRO, Canberra, 2018.

HunG, Walter, et al., "Managing the risk of overusing mobile phones in the working environment: a study of ubiquitous techno-stress", PACIS 2011 Proceedings Paper 81, 2011.

LiNDSAY, Greg, "We spent two weeks wearing employee Trackers: Here's what we learned", Fact Coexist, 2015, https://www.fastcompany.com/3051324/we-spent-twoweeks-wearing-employee-trackers-heres-what-we-learned (Acceso: 16/04/21).

MALGIERI, Gianclaudio, and COMAndÉ, Giovanni, "Why a Right to Legibility of Automated Decision Making Exists in the General Data Protection Regulation", International data privacy Law, vol. 7, nº 4, 2017, 243-265.

MendozA, Isak, and Bygrave, Lee, "The Right Not to Be Subject to Automated Decisions Based on Profiling", en Tatiani Synodinou et al. (eds.,) EU internet law: regulation and enforcement, 2017, https://papers.ssrn.com/abstract=2964855 [https://perma.cc/XV3T-G98W].

Mercader Uguina, Jesús, Nuevos escenarios para el Estatuto de los Trabajadores del siglo XXI. digitalizacción y cambio tecnológico”, TyD, nº 63, 2020.

Molina NAVARRete, Cristóbal, "La "Ley" de personas repartidoras en plataformas online ("riders”): ¿pequeño paso legal, gran paso para humanizar el precariado digital?”, Transformaework, 2021.

Molina NAVARRETE, Cristobal, Datos y derechos digitales de las personas trabajadoras en tiempos de (pos)covid19: entre eficiencia de gestión y garantías, Bomarzo, 2021.

Monereo PÉREz, José Luis y MArTín MuÑoz, María Rosa, "La laboralidad de quienes prestan servicios a través de plataformas digitales", Revista de Jurisprudencia Laboral, $n^{\circ} 9,2020$.

MoORE, Phoebe, Data subjects, digital surveillance, AI and the future of work, European Parliament Science and Technology Office, Brussels, 2020.

MOORE, Phoebe, Humans and machines at work: monitoring, surveillance and automation in contemporary capitalism, London. Palgrave Macmillan, 2018. 
MOORE, Phoebe, The quantified self in precarity. Work, technology and what counts, New York, Routledge, 2018.

MOORE, Phoebe, Work and the GDPR: The future for algorithms and people analytics Available at https://staffblogs.le.ac.uk/management/2018/09/20/work-and-the-gdpr-thefuture-for-algorithms-and-people-analytics/.

MoREno I GENÉ, Josep, "El carácter laboral de la prestación de servicios a través de plataformas digitales. el caso de Glovo. Comentario de la Sentencia del Tribunal Superior de Justicia de Cataluña de 21 de febrero de 2020", Revista General de Derecho del Trabajo y de la Seguridad Social, $\mathrm{n}^{\circ}$ 57, 2020.

MoReno i GEnÉ, Josep, "El carácter laboral de la prestación de servicios de reparto a través de la plataforma digital Glovo el Tribunal Supremo zanja el debate", IUSLabor, $\mathrm{n}^{\circ}$ 3, 2020 .

NiETo RojAs, Patricia, "Nuevamente sobre la laboralidad del vínculo contractual de los prestadores de servicios en plataformas digitales", Iuslabor, nº 1, 2019.

OIT, The role of digital labour platforms in transforming the world of work, Ginebra, 2021.

PAZOS PÉREZ, Alexandre, "Sobre el empleo en plataformas digitales. Comentario de la sentencia del Juzgado de lo Social núm. 1 de Madrid, de 4 de abril de 2019", Nueva revista española de derecho del trabajo, $\mathrm{n}^{\circ} 224,2019$, p. 247-256.

PÉRez De Los Cobos Orihuel, Francisco, "El trabajo subordinado como tipo contractual”, Documentación Laboral, nº 39, 1993.

PÉREZ DEL PRADO, Daniel, “La Ley Rider: un muy buen comienzo”, Foro de labos, 2021.

PÉREZ ZAPATA, Oscar, et al., "Digitalización, intensificación del trabajo y salud de los trabajadores españoles", 2019, (disponible en https://www.ccoo.es/24c0e370fa4b4d1f3682b1780854af9c000001.pdf. Acceso 29/04/2021).

PÉREZ ZAPATA, Oscar, Trabajo sin límites, salud insostenible: La intensificación del trabajo del conocimiento (e-prints, Universidad Complutense de Madrid), 2015. 
Pesole, Anna Rosa, Urzí Brancati, Maria Cesira, Fernández-Macías, Enrique, BIAgI, Federico y GonZÁlez VÁzQueZ, Ignacio, Platform Workers in Europe, EUR 29275 EN, Publications Office of the European Union, Luxembourg, 2018.

RodRÍGUEZ FERNÁNDEZ, María Luz, Protección social para los trabajadores de la economía de plataforma propuestas para aliviar su vulnerabilidad", Revista General de Derecho del trabjo y de la Seguridad Social, 57, 2020.

RODRÍGUEZ RoDRÍGUEZ, Emma, "La transcendencia de la disponibilidad horaria del trabajador en el contexto de las plataformas digitales", Temas Laborales, n 146, 2019, p. 121-158.

RodríGueZ-PiÑERo Royo, Miguel, “Por fin, la ley Rider”, en Trabajo, persona, Derecho y mercado, 2021, disponible en http://grupo.us.es/iwpr/2021/05/13/por-fin-la-ley-rider/

RODRÍGUEZ-RICO, Roldan, "Los retos para la prevención de riesgos laborales ante la tecnificación del trabajo", in Cerejeria Namora et al., Health at work, ageing and environmental effects on future social security and labour law Systems. Cambridge Scholars Publishing, 2018.

SAla Franco, Tomás y LóPEz Mora, Federico., "Contrato de trabajo", Comentarios a las leyes laborales. El Estatuto de los Trabajadores, Tomo I, 1990.

SÁNCHEZ-URÁN AZAÑA, Yolanda, "Concepto de trabajador y economía digital. Respuesta en el contexto internacional y en el derecho de la UE", Revista de Derecho Social y Empresa, $\mathrm{n}^{\circ}$ 14, 2021.

SANGUINETTI, Wilfredo, "La presunción de laboralidad de los riders o cómo se consiguió la cuadratura del círculo de la laboralidad”, El blog de Wilfredo Sanguinetti, 2021.

SCHUMACHER, Susan, "What employees should know about electronic performance monitoring”, ESSAI, vol. 8, n 28, 2011, p. 138-144.

SElBST, Andrew, and Powles, Julia, "Meaningful information and the right to explanation", International Data Privacy Law, n 233, 2017.

SuÁrez Corujo, Borja, "La gran transición: la economía de plataformas digitales y su proyección en el ámbito laboral y de la seguridad social”, Temas Laborales, nº 141, 2018, p. 37-66. 
Todolí Signes, Adrián, “Argumentos de la sanción a Cabify por cesión ilegal de la Inspección de trabajo -aplicables a muchas de las plataformas digitales-", Argumentos en Derecho laboral, 2021, disponible en https://adriantodoli.com/2021/03/25/argumentosde-la-sancion-a-cabify-por-cesion-ilegal-de-la-inspeccion-de-trabajo-aplicables-amuchas-de-las-plataformas-digitales/

Todolí Signes, Adrián, "Comentario a la Sentencia del Tribunal Supremo español que considera a los Riders empleados laborales.", Labour Law \& Issues, 6, 2, 2020, disponible en https://adriantodoli.com/2020/09/30/comentario-a-la-sentencia-del-tribunal-supremosobre-los-falsos-autonomos-en-glovo/

Todolí Signes, Adrián, "El ámbito subjetivo de aplicación del Estatuto de los Trabajadores ante las nuevas formas de trabajo: la nueva ley de California (AB5)", TyD, $n^{\circ} 66,2020$.

Todolí Signes, Adrián, "El concepto de trabajador en el Derecho de la Unión Europea y su aplicación a las nuevas realidades económicas: Comentario al auto motivado del TJUE en el caso de los riders", $T y D, \mathrm{n}^{\circ} 70,2020$.

Todolí SigneS, Adrián, "El Impacto de la "Uber economy" en las relaciones laborales los efectos de las plataformas virtuales en el contrato de trabajo", iuslabor, 2015, disponible para descargar en https://adriantodoli.com/2015/12/22/el-impacto-de-la-ubereconomy-en-las-relaciones-laborales-los-efectos-de-las-plataformas-virtuales-en-elcontrato-de-trabajo-2/

Todolí Signes, Adrián, "En cumplimiento de la primera Ley de la robótica: Análisis de los riesgos laborales asociados a un algoritmo artificial / inteligencia dirigiendo el trabajo", Labor Law \& Issues, vol. 5, $\mathrm{n}^{\mathrm{o}}$ 2, 2019, disponible en https://papers.ssrn.com/sol3/papers.cfm?abstract_id=3506584

TODOLí Signes, Adrián, “La evaluación de los trabajadores por parte de los clientes como método de vigilancia y control en la empresa: reputación online y protección de datos", CEF.RTSS, n 427,2018 , p. 63-90.

Todolí Signes, Adrián, "La gobernanza colectiva de la protección de datos en las relaciones laborales: big data, creación de perfiles, decisiones empresariales automatizadas y los derechos colectivos", $R D S, \mathrm{n}^{\circ} 84,2018$, p. 69-88.

Todolí SIGNES, Adrián, "Plataformas digitales y concepto de trabajador: una propuesta de interpretación finalista”, Lan harremanak, nº 41, 2019. 
TODOLÍ Signes, Adrián, El trabajo en la era de la economía colaborativa, Tirant lo Blanch, 2017.

Trillo PÁRragA, Francisco, "Calificación jurídica del trabajo en plataformas digitales. A propósito de la SJSOC 128/19, de 3 de abril", Nueva revista española de derecho del trabajo, no 224, 2019, p. 257-266.

Urzì Brancati, Cesira., Pesole, Anna Rosa., Fernández-Macías, Enrique. New evidence on platform workers in Europe. Results from the second COLLEEM survey, EUR 29958 EN, Publications Office of the European Union, Luxembourg, 2020.

UTS, Heat Stress and On-Demand work: The experience of food delivery and courier cyclists. Climate Justice Centre. 2019. Dosponible en https://opus.lib.uts.edu.au/bitstream/10453/134736/1/On\%20Demand\%20cyclists\%20U TS\%20final.pdf

Wachter, Sandra, Mittelstadt, Brent y Floridi, Luciano, "Why a Right to Explanation of Automated Decision-Making Does Not Exist in the General Data Protection Regulation” International Data Privacy Law, nº 7, 2017, p. 79-90.

WACHTER, Sandra., et al., "Counterfactual explanations without opening the black box: automated decisions and the GDPR", Arxiv, 2017, p. 1-51, en https://arxiv.org/ftp/arxiv/papers/1711/1711.00399.pdf

Wan, Kim, Routledge, Bernad, "Algorithmic Transparency, a Right to Explanation, and Placing Trust”, Squarespace, 2017, https://static1.squarespace.com/static/592ee286d482e908d35b8494/t/59552415579fb3 0c014cd06c/1498752022120/Algorithmic+transparency\%2C+a+right+to+explanation+ and+trust+\%28TWK\%26BR\%29.pdf [https://perma.cc/K53W-GVN2， (acceso: 07/05/2021. 